\title{
Degenerate Elastic Networks
}

\author{
Giacomo Del Nin ${ }^{1}(1) \cdot$ Alessandra Pluda $^{2}$ (I) $\cdot$ Marco Pozzetta $^{2}$ (1)
}

Received: 4 February 2020 / Accepted: 15 September 2020 / Published online: 9 October 2020

(c) The Author(s) 2020

\begin{abstract}
We minimize a linear combination of the length and the $L^{2}$-norm of the curvature among networks in $\mathbb{R}^{d}$ belonging to a given class determined by the number of curves, the order of the junctions, and the angles between curves at the junctions. Since this class lacks compactness, we characterize the set of limits of sequences of networks bounded in energy, providing an explicit representation of the relaxed problem. This is expressed in terms of the new notion of degenerate elastic networks that, rather surprisingly, involves only the properties of the given class, without reference to the curvature. In the case of $d=2$ we also give an equivalent description of degenerate elastic networks by means of a combinatorial definition easy to validate by a finite algorithm. Moreover we provide examples, counterexamples, and additional results that motivate our study and show the sharpness of our characterization.
\end{abstract}

Keywords Networks $\cdot$ Relaxation $\cdot$ Elastic energy $\cdot$ Singular structures

Mathematics Subject Classification Primary 49J45 - 35A15; Secondary 49Q10 . 53A04

\section{Introduction}

A regular network $\mathcal{N}$ is a connected set in $\mathbb{R}^{d}$ composed of $N$ regular curves $\gamma^{i}$ of class $H^{2}$ that meet at their endpoints in junctions of possibly different order. Moreover the angles at the junctions are assigned by a fixed set of directions $\mathcal{D}$ as we will define

Alessandra Pluda

alessandra.pluda@unipi.it

Giacomo Del Nin

Giacomo.Del-Nin@warwick.ac.uk

Marco Pozzetta

pozzetta@mail.dm.unipi.it

1 Mathematics Institute, University of Warwick, Zeeman Building, Coventry CV4 7HP, UK

2 Dipartimento di Matematica, Università di Pisa, Largo Bruno Pontecorvo 5, 56127 Pisa, Italy 
more precisely in Definition 2.11. The elastic energy functional $\mathcal{E}$ for a network $\mathcal{N}$ is given by

$$
\mathcal{E}(\mathcal{N}):=\sum_{i=1}^{N}\left(\int_{\gamma^{i}}\left|\vec{k}^{i}\right|^{2} \mathrm{~d} s+\ell\left(\gamma^{i}\right)\right)
$$

where $\vec{k}^{i}$ is the curvature, $s$ the arclength parameter and $\ell\left(\gamma^{i}\right)$ is the length of the curve $\gamma^{i}$

The elastic energy functional has a long history. Already at the times of Galileo scientists tried to model elastic rods and strings, looking for equations for equilibrium of moments and forces. The idea to relate the curvature of the fiber of the beam to the bending moment came only later when, in 1691, Jacob Bernoulli proposed to model the bending energy of thin inextensible elastic rods with a functional involving the curvature. Several authors refer to the functional (1.1) as Euler Elastic energy in honor of Euler (while we will simply call it elastic energy) who solved the problem of minimizing the potential energy of the elastic laminae using variational techniques. Even nowadays the elastic energy appears in several mechanical and physical models (c.f. [21]) and in imaging sciences, see for instance [18].

We are interested in the minimization of the functional $\mathcal{E}$ among networks with fixed topology and with fixed angles at the junctions assigned by $\mathcal{D}$.

Notice that the length $\ell\left(\gamma^{i}\right)$ of each curve of a regular network is strictly positive because the curves are regular by assumption. This property is not preserved by sequences with uniformly bounded energy: along a sequence of networks $\left\{\mathcal{N}_{n}\right\}_{n \in \mathbb{N}}$ with uniformly bounded energy (or even a minimizing sequence) the length of a curve may go to zero as $n \rightarrow \infty$, producing a "degenerate limit" which is no longer a regular network. Hence a remarkable issue is to understand this lack of compactness of sequences with bounded energy in minimization problems. ${ }^{1}$

Our first task is therefore to describe the class of limits of sequences (equibounded in energy) of regular networks. In other words, we have to characterize the "closure in energy" of the class $\mathcal{C}_{\text {Reg }}$ of regular networks.

Then, since the lack of compactness implies that it could not be possible to solve the original minimization problem among regular networks, we relax it by considering the lower semicontinuous envelope of the functional $\mathcal{E}$ with respect to the weak convergence in $H^{2}$. Our second objective is to find an explicit formula of the lower semicontinuous envelope. We underline that the relaxation of the problem is necessary: Example 5.2 shows that also in some very simple situations minimizers are not regular networks.

In Propositions 3.9 and 4.2 we characterize the smallest compact class of (nonregular) networks in which the class of regular networks is "dense in energy" in terms of a mixture of algebraic and combinatorial conditions that are easy to verify and rely on the topological assumptions on the competitors. As the formulation of these conditions involves some technicalities, a detailed description is postponed to the second part of the introduction. We are then able to give a characterization of the

\footnotetext{
1 By compactness we mean sequential compactness, where the natural notion of convergence for the sequences of networks is the weak $H^{2}$ convergence.
} 
Fig. 1 A Theta-network and a degenerate Theta-network
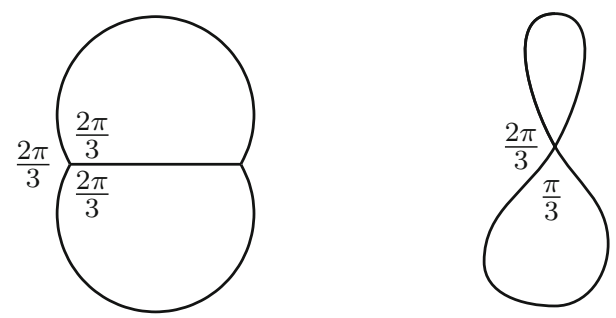

lower semicontinuous envelope of $\mathcal{E}$ that strongly relies on the previous result (see Theorem 4.8).

This last point creates a bridge between our problem and another natural research direction: the extension of the elastic energy (and more in general of functionals that depend on the curvature) to singular sets for which at least a weak notion of curvature is well defined, varifolds for instance. The characterization of the relaxation of the original functional in such cases turned out to be particularly difficult (for results in this direction see for instance $[4-7,15,16,19]$ ). We notice that networks can be seen as a simple example of sets which are essentially singular and they can be understood as a boundary of a planar cluster of surfaces. For problems in which one knows a priori that the boundary of minimal planar clusters is composed of a finite number of curves, it could be useful to define the curvature of the cluster by means of (1.1). An advantage is that we have an easy integral representation of the lower semicontinuous envelope of $\mathcal{E}$.

To place our paper in the broader context, we mention the minimization of Willmoretype functionals among both Riemannian manifolds and singular structures is an extreme flourishing research field. As the simplest possible example of singular structure we mention surfaces with boundary (possible references are [2,9-11,17,20]).

The main reason for which we got interested in this problem is a previous study of its dynamical counterpart (see $[13,14]$ ). The study of the static problem has often revealed to be useful for the analysis of the asymptotic behavior of the solutions of the associated gradient flow and of the singularities that can appear during the evolution. Our analysis can be useful to understand the long time behavior of the elastic flow of networks whose curves meet at junctions with prescribed angles introduced in [3,14].

After this detour on the literature, we explain more in detail what can happen along sequences of regular networks $\left\{\mathcal{N}_{n}\right\}_{n \in \mathbb{N}}$ whose elastic energy is uniformly bounded.

\section{A Model Problem: Theta-Networks}

A Theta-network is a regular 3-network whose curves form equal angles at the two junctions (Fig. 1). The limit of a sequence of regular Theta-networks with uniformly bounded energy may not exist in the class of regular networks and hence the minimization problem has some form of degeneracy that luckily is not completely wild: the length of at most one curve can go to zero along the sequence, becoming straighter and straighter. If this is the case the "degenerate" limit network is composed of two curves meeting at a quadruple point forming angles equal in pairs of $\frac{\pi}{3}$ and $\frac{2 \pi}{3}$.

The general case presents new interesting features with respect to the model problem of Theta-networks. Firstly, one realizes that much more than the length of one single curve can go to zero, since entire parts of the network can vanish as $n \rightarrow \infty$. In order 


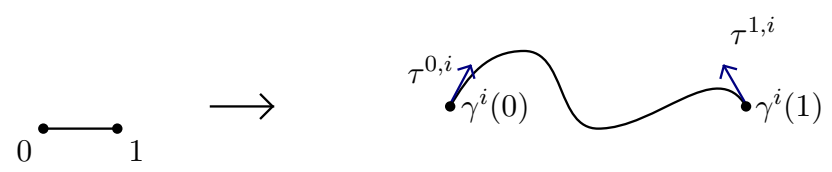

Fig. 2 Outer tangents at the endpoints of the curve $\gamma^{i}$

to keep track of the complexity of the network we introduce the notion of underlying graph $G$.

\section{The Underlying Graph}

The oriented graph $G$ is composed of edges $E_{i}$ whose endpoints are identified in vertices of possibly different order (Definition 2.1). A network then is a pair graphcontinuous map $\mathcal{N}=(G, \Gamma)$ with $\Gamma: G \rightarrow \mathbb{R}^{d}$ (Definition 2.2). The underlying graph $G$ captures the topology of the networks and allows us to have a reminiscence of their structure even when some curves in a sequence of networks $\mathcal{N}_{n}=\left(G, \Gamma_{n}\right)$ collapse to a point in the limit.

\section{A Necessary Angle Condition}

We say that a curve $\gamma^{i}:=\left.\Gamma\right|_{E_{i}}$ of a network is singular if it is a constant map, and regular if it is an immersion of class $H^{2}$. A network is singular if some of its curves are singular.

Definition 1.1 (Outer tangents) Consider a regular curve of a network $\mathcal{N}$ parametrized by $\gamma^{i}$. We define $\tau^{z, i}$, with $z \in\{0,1\}$, as the outer tangent vector at the endpoint $\gamma^{i}(z)$ to the curve $\gamma^{i}$ given by

$$
\tau^{z, i}=(-1)^{z} \frac{\dot{\gamma}^{i}(z)}{\left|\dot{\gamma}^{i}(z)\right|} .
$$

We remark that the vector $\tau^{z, i}$ "points inside" the curve $\gamma^{i}$ at $\gamma^{i}(z)$ (see Fig. 2). In this way, if $\gamma^{i}\left(z_{i}\right)=\gamma^{j}\left(z_{j}\right)$ is a junction point, then $\tau^{z_{i}, i}$ and $\tau^{z_{j}, j}$ "point outwards" with respect to the junction. In this way the outer tangents are geometrically independent of the direction of the parametrization of the curves concurring at a junction.

The following definition introduces the angle condition for a possibly singular network. It will be restated more in detail in Definition 3.2.

Definition 1.2 (Angle condition) A (possibly) singular network $\mathcal{N}=(G, \Gamma)$ satisfies the angle condition if for every singular curve $\gamma^{i}$ there exist two unit vectors $\tau^{z, i}$, called its virtual tangents, with $z \in\{0,1\}$ such that $\tau^{0, i}=-\tau^{1, i}$ and such that, at each junction, the tangent vectors (either real outer tangents or virtual ones) coincide, up to a fixed rotation that only depends on the junction, with the directions assigned by a given set $\mathcal{D}$ (we shall state this concept more in detail in Definitions 2.11 and 3.2).

Also motivated by [8], as a first attempt we defined the class of degenerate networks as all the singular networks that satisfy the angle condition of Definition 1.2.

Unfortunately this purely algebraic condition on the angles turns out to be necessary but not sufficient to characterize the closure of the class $\mathcal{C}_{\text {Reg }}$ as we show in the next example. 
Fig. 3 Starting from the represented graph $G$, it is possible to construct an example from which one deduces that the angle condition is not sufficient to define the closure of the class $\mathcal{C}_{\text {Reg. }}$. See Example 3.4 for details

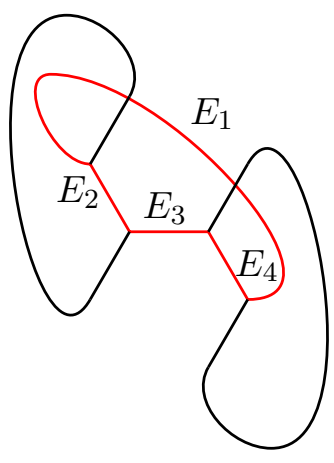

Consider the topology depicted in Fig. 3 on the left, with all junctions forming angles of $\frac{2 \pi}{3}$, and define a singular network that collapses the red part to a single point. It is possible to do so satisfying the angle condition of Definition 1.2 (see the picture on the right) but no sequence of regular networks with finite energy and the same angle constraint could converge to the singular network on the right.

Indeed, denoting by $C$ the cycle in red in Fig. 3, using Gauss-Bonnet Theorem (Remark 3.3) one gets $\mathcal{E}(C) \geq \frac{c}{L(C)}$, with $c>0$, for any immersion $\Gamma$ such that $(G, \Gamma)$ is a regular network, and thus the energy diverges if the length of the red loop goes to zero (see Example 3.4 and Remark 3.3 for more details).

\section{Stratified Straight Subgraph}

To overcome this issue in the definition of the class, we looked for an extra condition. Again the underlying graph $G$ helps us.

Definition 1.3 (Stratified straight subgraph) A subgraph $H$ of the underlying graph $G$ is stratified-straight if there exists a finite sequence of subgraphs, called strata,

$$
\emptyset=H_{q} \subset H_{q-1} \subset \ldots \subset H_{1} \subset H_{0}=H
$$

and maps $\Sigma_{j}: H_{j} \rightarrow \mathbb{R}^{d}$ such that for $j=0, \ldots, q-1$

- the (sub)network $\left(H_{j}, \Sigma_{j}\right)$ satisfies the angle condition 1.2 with (real or virtual) tangent vectors coinciding with the ones associated to $\left(H_{0}, \Sigma_{0}\right)$ and its regular curves are straight segments;

- the stratum $H_{j+1}$ is the union of the singular curves of $\left(H_{j}, \Sigma_{j}\right)$.

In the following, segments are always understood to be straight segments.

Denote by $H$ the subgraph given by the union of the edges $E_{i}$ of $G$ such that the curves $\gamma^{i}=\left.\Gamma\right|_{E_{i}}$ are singular. With a little abuse of notation we say that the network $\mathcal{N}=(G, \Gamma)$ is stratified straight if $H$ is stratified straight.

\section{The Class of Degenerate Networks}

Finally we come to the characterization: a network is degenerate, of class $\mathcal{C}_{\text {Deg }}$, if it satisfies the angle condition 1.2 and it is stratified straight. It is natural now to define the extension $\overline{\mathcal{E}}$ of the functional $\mathcal{E}$ by setting that a curve with zero length of 
a network in $\mathcal{C}_{\text {Deg }}$ gives a null contribution to the energy and by assigning value $+\infty$ to the energy of singular networks which are not in $\mathcal{C}_{\text {Deg }}$.

In Proposition 3.9 we prove that a sequence of regular networks with equibounded energy converges to a network in $\mathcal{C}_{\text {Deg }}$, namely that the closure of $\mathcal{C}_{\text {Reg }}$ is contained into $\mathcal{C}_{\text {Deg. }}$.

\section{Formula for the Lower Semicontinuous Envelope of the Elastic Energy Func- tional}

A question still remains: is the class $\mathcal{C}_{\text {Deg }}$ the smallest set of generalized network where we have compactness? In Proposition 4.2 we show that any degenerate network can be approximated in energy by a regular one. Hence our guess was correct: the class $\mathcal{C}_{\text {Reg }}$ is "dense in energy" in $\mathcal{C}_{\text {Deg }}$. Moreover the extension $\overline{\mathcal{E}}$ is the lower semicontinuous envelope of $\mathcal{E}$.

We stress the fact that we are able to express the lower semicontinuous envelope in integral form and with the very simple formula (4.1). This is possible only thanks to the precise characterization of $\mathcal{C}_{\text {Deg }}$.

Notice that the existence of minimizers of the relaxed functional $\overline{\mathcal{E}}$ follows trivially by a direct method in the Calculus of Variations.

\section{Stratified Straight Versus Straight Subgraph}

At first sight considering stratified straight subgraphs could seem uselessly complicated.

One can ask whether the class of degenerate networks can be equivalently characterized as the set of networks $(G, \Gamma)$ satisfying the angle condition 1.2 such that there exists only one map $\Sigma: H \rightarrow \mathbb{R}^{d}$ from the singular edges to $\mathbb{R}^{d}$ such that every curve of $\Sigma$ is a regular straight segment and $(H, \Sigma)$ satisfies the angle condition (in this case we say that the network $(H, \Sigma)$ is straight). This would mean that in Definition 1.3 the index $q=1$ is enough. In Example 3.10 we show a stratified straight but not straight graph. Nonetheless, there are some cases in which every stratified straight subgraph is in fact straight; an easy example consists in graphs $G$ with the structure of a tree.

However, the concepts of stratified straight graphs and straight graphs are essentially different, even in non-trivial cases that have some interest from a variational viewpoint; more precisely in Sect. 7 we study the case of networks in $\mathbb{R}^{2}$ with graphs having junctions of order at most four such that at these junctions the tangent vectors are orthogonal (Proposition 7.5) and we find a characterization of the stratified straight graphs that turn out to be straight.

\section{Ambient Space: Restriction to the Plane and Extension to Riemannian Surfaces}

Although we find our characterization of the class $\mathcal{C}_{\text {Deg }}$ by combination of algebraic and geometric conditions quite satisfactory, if the ambient space is $\mathbb{R}^{2}$ we are able to give an equivalent characterization of the class $\mathcal{C}_{\text {Deg }}$ (see Definition 3.8) by an algebraic-topological condition that has the great advantage of being verifiable by an algorithm with finitely many steps.

It is also worth to mention that our result extends from the ambient space $\mathbb{R}^{2}$ to any 2-dimensional closed surface embedded in $\mathbb{R}^{3}$. 


\section{Fixed Lengths}

In Sect. 8.2 we present a variant of the problem, that is analytically very simple, but that could be more relevant from the physical point of view: the minimization of the $L^{2}$-norm of the curvature among networks whose curves have fixed length.

\section{Plan of the Paper}

The structure of the paper is the following: after the definitions of networks and elastic energy contained in Sect. 2, in Sect. 3 we introduce the class of degenerate networks (Definition 3.8). The compactness of the class of degenerate networks is proved in Proposition 3.9. In Sect. 4 we finish the proof of the representation of the relaxed functional by constructing recovery sequences of degenerate networks (Proposition 4.2). Section 6 is devoted to the equivalent algorithmic characterization of the class of degenerate networks when Problem 2.13 is set in $\mathbb{R}^{2}$. Then in Sect. 7 we study a non-trivial case in which there exist stratified straight subgraphs that are not straight and we characterize such difference (Proposition 7.5). Subsequently we comment on the extension of our result from the ambient space $\mathbb{R}^{2}$ to any 2-dimensional closed surface embedded in $\mathbb{R}^{3}$. In Sect. 8 we collect some final observations and remarks. We conclude the paper with the Appendix A where we compute the EulerLagrange equations satisfied by critical points of the energy and we prove that critical points are real analytic, up to reparametrization.

\section{Setting and Definition of the Problem}

We begin by defining the mathematical objects of our interest.

\subsection{Elastic Energy Functional for Networks}

Fix $N \in \mathbb{N}, d \in \mathbb{N}$ with $d \geq 2$ and let $i \in\{1, \ldots, N\}, I_{i}:=[0,1] \times\{i\}, E:=\bigcup_{i=1}^{N} I_{i}$ and $V:=\bigcup_{i=1}^{N}\{0,1\} \times\{i\}$.

Definition $2.1(N$-graph $)$ Let $\sim$ be an equivalence relation that identifies points of $V$. An $N$-graph $G$ is the topological quotient space of $E$ induced by $\sim$, that is

$$
G:=E / \sim
$$

A subgraph $H \subseteq G$ is the quotient

$$
H:=\bigcup_{j=1}^{M} I_{i_{j}} / \sim
$$

for a given choice of indices $i_{1}, \ldots, i_{M} \in\{1, \ldots, N\}$, where $\sim$ is the equivalence relation defining $G$ (Fig. 4).

We notice that the natural projection $\pi: E \rightarrow G$ restricted to $E \backslash V$ is a homeomorphism with its image. 

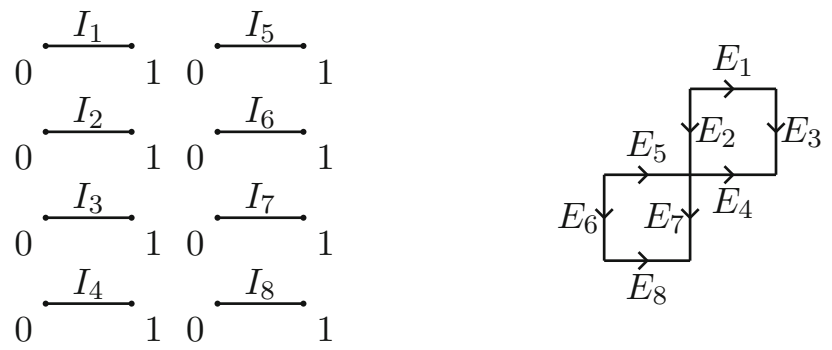

Fig. 4 On the left an example of a set $E$ and on the right the resulting graph $G$ with the identifications: $(0,1) \sim(0,2),(1,1) \sim(0,3),(1,2) \sim(0,4) \sim(1,5) \sim(0,7),(1,3) \sim(1,4),(0,5) \sim(0,6)$, $(1,6) \sim(0,8),(1,7) \sim(1,8)$

We call

$$
E_{i}:=I_{i} / \sim, \quad \text { and } \quad V_{G}:=V / \sim
$$

Definition 2.2 [N-network] An $N$-network (or simply a network) is a pair $\mathcal{N}=(G, \Gamma)$ where

$$
\Gamma: G \rightarrow \mathbb{R}^{d}
$$

is a continuous map and $G$ is an $N$-graph. Moreover we ask each $\gamma^{i}:=\Gamma_{\mid E_{i}}$ to be either a constant map (singular curve) or an immersion of class $H^{2}$ (regular curve).

We will identify two networks $\left(G_{1}, \Gamma_{1}\right)$ and $\left(G_{2}, \Gamma_{2}\right)$ if $G_{1}=G_{2}=G$ and for any edge $E_{i}$ of $G$ the curve $\left.\Gamma_{1}\right|_{E_{i}}$ coincides with $\left.\Gamma_{2}\right|_{E_{i}}$ up to reparametrization.

Definition 2.3 (Convergence of networks) We define that a sequence of networks $\mathcal{N}_{n}=$ $\left(G_{n}, \Gamma_{n}\right)$ converges to a limit network $\mathcal{N}_{\infty}=\left(G_{\infty}, \Gamma_{\infty}\right)$ in a certain topology if $G_{n}=G_{\infty}$ for any $n$ and each sequence of curves $\left.\Gamma_{n}\right|_{E_{i}}$ converges to $\left.\Gamma_{\infty}\right|_{E_{i}}$, up to reparametrization, in that topology.

Definition 2.4 (Singular part) Given a network $\mathcal{N}=(G, \Gamma)$ we define its singular part $\operatorname{Sing}(\mathcal{N})$ as the subgraph $H \subset G$ whose edges are exactly those associated to singular curves of $\mathcal{N}$ (mapped by $\Gamma$ to a point) and we call regular $\operatorname{part} \operatorname{Reg}(\mathcal{N}):=$ $\overline{G \backslash \operatorname{Sing}(\mathcal{N})}$.

If $E_{i} \subset \operatorname{Reg}(\mathcal{N})$ has a common vertex $p$ with $E_{j} \in \operatorname{Sing}(\mathcal{N})$ we say that $p$ belongs to $\operatorname{Sing}(\mathcal{N})$, i.e., $\operatorname{Sing}(\mathcal{N})$ is closed.

Definition 2.5 (Regular and singular networks) We say that a network $\mathcal{N}=(G, \Gamma)$ is regular if $\operatorname{Sing}(\mathcal{N})=\emptyset$ and singular otherwise.

Definition 2.6 Given a network $\mathcal{N}$ we denote by $\ell\left(\gamma^{i}\right)$ the length of the curve $\gamma^{i}$. The length of the network $\mathcal{N}$ is nothing but

$$
L(\mathcal{N}):=\sum_{i=1}^{N} \ell\left(\gamma^{i}\right) .
$$


Consider a regular curve $\gamma^{i}$ of a network $\mathcal{N}$. Then we define its curvature vector as the $L^{2}$ function given by

$$
\vec{k}^{i}=\frac{\ddot{\gamma}^{i}}{\left|\dot{\gamma}^{i}\right|^{2}}-\frac{\left\langle\ddot{\gamma}^{i}, \dot{\gamma}^{i}\right\rangle \dot{\gamma}^{i}}{\left|\dot{\gamma}^{i}\right|^{4}}
$$

Observe that by the Sobolev embedding $\dot{\gamma}$ is Hölder continuous, and thus by the regularity its norm is bounded and bounded away from zero.

We are now in position to introduce the functional of our interest.

Definition 2.7 Let $\alpha, \beta>0$. The elastic energy functional $\mathcal{E}_{\alpha, \beta}$ for a regular network $\mathcal{N}$ is defined as

$$
\mathcal{E}_{\alpha, \beta}(\mathcal{N}):=\alpha \int_{\mathcal{N}}|\vec{k}|^{2} \mathrm{~d} s+\beta L(\mathcal{N})=\sum_{i=1}^{N}\left(\alpha \int_{\mathcal{N}^{i}}\left|\vec{k}^{i}\right|^{2} \mathrm{~d} s^{i}+\beta \ell\left(\gamma^{i}\right)\right)
$$

where $\vec{k}^{i}$ is the curvature and $s^{i}$ is the arclength parameter.

Remark 2.8 Reparametrizing the regular curves of the network with constant speed equal to the length we can write (2.1) as

$$
\mathcal{E}(\mathcal{N})=\sum_{i=1}^{N}\left(\alpha \int_{0}^{1} \frac{\left|\ddot{\gamma}^{i}(x)\right|^{2}}{\left(\ell\left(\gamma^{i}\right)\right)^{3}} \mathrm{~d} x+\beta \ell\left(\gamma^{i}\right)\right)
$$

By assigning to each singular curve of a network a zero curvature $\vec{k}^{i}=\overrightarrow{0}$ we can naturally extend $\mathcal{E}$ to the class of all networks maintaining exactly the same formula. The elastic energy $\widetilde{\mathcal{E}}_{\alpha, \beta}$ of a regular or singular network $\mathcal{N}$ is thus

$$
\widetilde{\mathcal{E}}_{\alpha, \beta}(\mathcal{N}):=\alpha \int_{\mathcal{N}}|\vec{k}|^{2} \mathrm{~d} s+\beta L(\mathcal{N}),
$$

where $\vec{k}^{i}$ is the curvature of the regular curves of $\mathcal{N}$ or the assigned zero curvature on the singular curves of $\mathcal{N}$.

\subsection{Definition of the Minimization Problem}

Definition 2.9 (Junction of order $m$ ) Consider an $N$-graph $G$ and $p \in V_{G}$. We say that $p$ is a junction of order $m$ (with $m \in\{1, \ldots, N\}$ ) if

$$
\sharp \pi^{-1}(p)=m,
$$

where $\pi$ is the projection defined below Definition 2.1 and $\sharp$ denotes the cardinality of a set. 
Definition 2.10 (Graph with assigned angles) We say that an $N$-graph $G$ has assigned angles if to every junction $p \in V_{G}$ we assign directions

$$
d^{z_{1}, i_{1}}, \ldots, d^{z_{m}, i_{m}} \in \mathbb{S}^{d-1}
$$

where $m$ is the order of the junction and where

$$
p=\pi\left(z_{1}, i_{1}\right)=\ldots=\pi\left(z_{m}, i_{m}\right),
$$

with $\left(z_{1}, i_{1}\right), \ldots,\left(z_{m}, i_{m}\right) \in\{0,1\} \times\{1, \ldots, N\}$ all distinct. We denote by $\mathcal{D}$ the set of all the assigned directions.

Definition 2.11 (Angle condition for a regular network) Given $G$ an $N$-graph with assigned angles, we say that the regular network $\mathcal{N}=(G, \Gamma)$ fulfills the angle condition if for every $p \in V_{G}$ junction of order $m$, writing

$$
p=\pi\left(z_{1}, i_{1}\right)=\ldots=\pi\left(z_{m}, i_{m}\right)
$$

with $\left(z_{1}, i_{1}\right), \ldots,\left(z_{m}, i_{m}\right) \in\{0,1\} \times\{1, \ldots, N\}$ all distinct, there exists a rotation $\mathrm{R}_{p}: \mathbb{R}^{d} \rightarrow \mathbb{R}^{d}$ depending on $p$ such that

$$
\tau^{z_{1}, i_{1}}=\mathrm{R}_{p}\left(d^{z_{1}, i_{1}}\right), \ldots, \tau^{z_{m}, i_{m}}=\mathrm{R}_{p}\left(d^{z_{m}, i_{m}}\right),
$$

where $\tau^{z, i}$ are the outer tangents introduced in Definition 1.1.

Definition 2.12 (Class $\mathcal{C}_{\text {Reg }}$ ) Fix an $N$-graph with assigned angles. We say that a network $\mathcal{N}=(G, \Gamma)$ belongs to $\mathcal{C}_{\text {Reg }}(\mathcal{D})$ if it is regular and fulfills the angle condition with the directions assigned by $\mathcal{D}$ in the sense of Definition 2.11 .

Problem 2.13 Given an $N$-graph $G$ with assigned angles by $\mathcal{D}$ we want to study

$$
\inf \left\{\mathcal{E}_{\alpha, \beta}(\mathcal{N}) \mid \mathcal{N}=(G, \Gamma) \in \mathcal{C}_{\operatorname{Reg}}(\mathcal{D})\right\}
$$

From now on, for sake of notation, we simply write $\mathcal{C}_{\text {Reg }}$ instead of $\mathcal{C}_{\text {Reg }}(\mathcal{D})$.

Remark 2.14 It is not restrictive to ask $G$ to be connected, otherwise one minimizes the energy of each connected component of $G$.

Remark 2.15 Let us call $\mathcal{E}:=\mathcal{E}_{1,1}$. The rescaling properties of the functional $\mathcal{E}_{\alpha, \beta}$ imply

$$
\mathcal{E}_{\alpha, \beta}(\mathcal{N})=\sqrt{\alpha \beta} \mathcal{E}\left(\sqrt{\frac{\beta}{\alpha}} \mathcal{N}\right)
$$

so if $\mathcal{N}_{\text {min }}$ is a minimizer for $\mathcal{E}$, then the rescaled network $\frac{\beta}{\alpha} \mathcal{N}_{\text {min }}$ is a minimizer for $\mathcal{E}_{\alpha, \beta}$ and vice versa. Hence it is not restrictive to fix $\alpha=\beta=1$. 
Remark 2.16 Given a graph $G$ with assigned angles, there always exists a map $\Gamma$ : $G \rightarrow \mathbb{R}^{d}$ such that $(G, \Gamma)$ is a regular network with finite energy. It is sufficient to send each vertex $p \in V_{G}$ to a point $x^{j}$ in $\mathbb{R}^{d}$ and to connect the points $x^{i}$ with curves of finite length and with bounded curvature whose outward tangent vectors are chosen accordingly with the fixed directions $d^{z_{j}, i_{j}}$. The class $\mathcal{C}_{\text {Reg }}$ is hence not empty and (2.4) is finite.

\section{Compactness}

With a little abuse of notation by considering a sequence of networks $\left\{\mathcal{N}_{n}\right\}_{n \in \mathbb{N}}$ we mean that we consider a sequence of pairs $\left(G,\left\{\Gamma_{n}\right\}_{n \in \mathbb{N}}\right)$ where the $N$-graph $G$ with assigned angles is fixed.

Lemma 3.1 Let $\left\{\mathcal{N}_{n}\right\}_{n \in \mathbb{N}}$ be a sequence of networks in $\mathcal{C}_{\text {Reg }}$ such that

$$
\limsup _{n} \mathcal{E}\left(\mathcal{N}_{n}\right) \leq C<+\infty
$$

Suppose that for a certain index $i \in\{1, \ldots, N\}$

$$
\lim _{n \rightarrow \infty} \ell\left(\gamma_{n}^{i}\right)=0
$$

then

$$
\lim _{n \rightarrow \infty} \sup _{x, y \in[0,1]}\left|\frac{\dot{\gamma}_{n}^{i}(x)}{\left|\dot{\gamma}_{n}^{i}(x)\right|}-\frac{\dot{\gamma}_{n}^{i}(y)}{\left|\dot{\gamma}_{n}^{i}(y)\right|}\right|=0 .
$$

Proof Since $\left\{\mathcal{N}_{n}\right\}_{n \in \mathbb{N}}$ is a sequence of regular networks, we can suppose (up to reparametrisation) that for every $n \in \mathbb{N}$, for every $i \in\{1, \ldots, N\}$ the immersion $\gamma_{n}^{i}:[0,1] \rightarrow \mathbb{R}^{2}$ is a regular parametrization with constant speed equal to its length. Given $x, y \in[0,1]$, we get

$$
\begin{aligned}
\left|\frac{\dot{\gamma}_{n}^{i}(x)}{\left|\dot{\gamma}_{n}^{i}(x)\right|}-\frac{\dot{\gamma}_{n}^{i}(y)}{\left|\dot{\gamma}_{n}^{i}(y)\right|}\right| & =\frac{1}{\ell\left(\gamma_{n}^{i}\right)}\left|\dot{\gamma}_{n}^{i}(x)-\dot{\gamma}_{n}^{i}(y)\right|=\frac{1}{\ell\left(\gamma_{n}^{i}\right)}\left|\int_{x}^{y} \ddot{\gamma}_{n}^{i}(t) \frac{\ell\left(\gamma_{n}^{i}\right)^{2}}{\ell\left(\gamma_{n}^{i}\right)^{2}} \mathrm{~d} t\right| \\
& \leq\left|\int_{\gamma_{n}^{i}}\right| \vec{k}_{n}^{i}|\mathrm{~d} s| \leq \mathcal{E}\left(\mathcal{N}_{n}\right)^{1 / 2} \ell\left(\gamma_{n}^{i}\right)^{1 / 2} \\
& \leq C \ell\left(\gamma_{n}^{i}\right)^{1 / 2} .
\end{aligned}
$$

We then obtain the desired result passing to the limit.

We state here again the angle condition 1.2 with the use of the notation introduced in Sect. 2.

Definition 3.2 (Angle condition for a singular network) Consider a (possibly singular) network $\mathcal{N}=(G, \Gamma)$. We recall that for any $p \in V_{G}$ and $\gamma^{i}$ regular curve such 
that $\pi(z, i)=p$ for some $z \in\{0,1\}$ the usual (real) outward tangent vector is $\tau^{z, i}=(-1)^{z} \frac{\dot{\gamma}^{i}(z)}{\left|\dot{\gamma}^{i}(z)\right|}$.

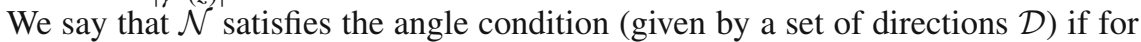
every singular curve $\gamma^{i}$ there exist unit vectors $\tau^{0, i}$ and $\tau^{1, i}$ (called virtual tangent vectors) such that $\tau^{0, i}=-\tau^{1, i}$ and such that for every $p \in V_{G}$ there exists a rotation $\mathrm{R}_{p}$ in $\mathbb{R}^{d}$ such that

$$
\tau^{z_{1}, i_{1}}=\mathrm{R}_{p}\left(d^{z_{1}, i_{1}}\right), \ldots, \tau^{z_{m}, i_{m}}=\mathrm{R}_{p}\left(d^{z_{m}, i_{m}}\right)
$$

where $\pi^{-1}(p)=\left\{\left(z_{1}, i_{1}\right), \ldots,\left(z_{m}, i_{m}\right)\right\}$ and $\tau^{z_{j}, i_{j}}$ are either the real outward tangent vectors or the virtual ones.

Remark 3.3 (Gauss-Bonnet-type theorems for curves in the plane) Let $\sigma:[a, b] \rightarrow$ $\mathbb{R}^{2}$ be an embedded piecewise $H^{2}$, regular closed curve, that is, there exist points $a=x_{0}<x_{1}<\ldots<x_{n-1}<x_{n}=b$ such that $\left.\sigma\right|_{\left[x_{j}, x_{j+1}\right]}$ is a regular curve of class $H^{2}, \sigma$ is continuous on $[a, b]$, and $\sigma(a)=\sigma(b)$. Denote by $\dot{\sigma}\left(x_{j}^{ \pm}\right):=\lim _{t \rightarrow x_{j}^{ \pm}} \dot{\sigma}(t)$, which exists as $\dot{\sigma}(t)$ is $\frac{1}{2}$-Hölder continuous on $\left[x_{j}, x_{j+1}\right]$ for $j=0, \ldots, n-1$. Finally denote by $\Theta[v, w] \in(-\pi, \pi]$ the angle between two vectors $v, w \in \mathbb{R}^{2}$, taken with positive sign if and only if the ordered couple $(v, w)$ is a positive basis of $\mathbb{R}^{2}$. Then the classical Gauss-Bonnet theorem (which follows for instance from Hopf's Umlaufsatz [1, Theorem 2.4.7]) reads

$$
\int_{a}^{b}\left\langle\vec{k}_{\sigma}, v_{\sigma}\right\rangle=2 \pi-\sum_{j=0}^{n-1} \Theta\left[\dot{\sigma}\left(x_{j}^{-}\right), \dot{\sigma}\left(x_{j}^{+}\right)\right],
$$

where $\vec{k}_{\sigma}$ is the curvature vector of $\sigma, v_{\sigma}$ is its normal vector, $\dot{\sigma}\left(x_{0}^{-}\right) \equiv \dot{\sigma}\left(a^{-}\right):=$ $\dot{\sigma}\left(b^{-}\right)$, and we are assuming that $\sigma$ positively parametrizes the boundary of the bounded connected component it encloses.

Below we will need a refined version of this theorem. Suppose now that the curve $\sigma$ has the same regularity as above but it is possibly immersed, i.e., it may have self-intersections, then the Gauss-Bonnet theorem (see [8, Theorem A.1]) reads

$$
\int_{a}^{b}\left|\vec{k}_{\sigma}\right| \mathrm{d} s \geq 2 \pi-\sum_{j=0}^{n-1}\left|\Theta\left[\dot{\sigma}\left(x_{j}^{-}\right), \dot{\sigma}\left(x_{j}^{+}\right)\right]\right| .
$$

We discuss here more in detail the example, anticipated in the Introduction, of a singular network that satisfies the angle condition 3.2 but that cannot be the limit of a sequence of regular networks with uniformly bounded energy.

Example 3.4 Let the dimension of the ambient space be $d=2$. Consider the 6-graph of Fig. 3 where in particular the edges $E_{1}, E_{2}, E_{3}, E_{4}$ form a cycle $C^{2}$ and, as sketched

\footnotetext{
${ }^{2}$ Namely the equivalence relation that defined $G$ is given by $(1, i) \sim(0, i+1)$ with the index $i$ considered modulo 4 .
} 
in the picture, assume that the assigned directions $\left\{d^{z, i}\right\}$ at the (triple) junctions form (equal) angles equal to $\frac{2}{3} \pi$. Consider a sequence of continuous maps $\left\{\Gamma_{n}\right\}_{n \in \mathbb{N}}$ such that $\Gamma_{1}$ is, for example, as depicted in Fig. 3 and such that $L\left(C_{n}\right) \rightarrow 0$, where $C_{n}:=\left.\Gamma_{n}\right|_{C}$. This property is satisfied by any sequence of networks approximating the singular network $\mathcal{N}_{\infty}=\left(G, \Gamma_{\infty}\right)$ depicted on the right in Fig. 3, which collapses the cycle $C$. Observe that there exists a choice of virtual tangents for which $\mathcal{N}_{\infty}$ satisfies the angle condition in the sense of Definition 3.2. But combining (3.2) and Hölder inequality, we get

$$
\mathcal{E}\left(\Gamma_{n}\right) \geq \mathcal{E}\left(C_{n}\right) \geq \int_{C_{n}}|\vec{k}|^{2} \mathrm{~d} s \geq \frac{\left(\int_{C_{n}}|\vec{k}| \mathrm{d} s\right)^{2}}{L\left(C_{n}\right)} \geq \frac{\left(2 \pi-4 \frac{\pi}{3}\right)^{2}}{L\left(C_{n}\right)}
$$

hence, as $n \rightarrow \infty$, the energy diverges as the length of the red loop $C_{n}$ goes to zero.

The angle condition for a singular network is thus not sufficient to characterize the set of limit networks with bounded energy.

Definition 3.5 (Straight graph) An $N$-graph $G$ with assigned angles is straight if there exists a regular network $\mathcal{N}=(G, \Gamma)$ whose curves are straight segments that fulfills the angle condition in the sense of Definition 2.11.

Suppose that $G$ is an $N$-graph with assigned angles. Then every subgraph $H$ of $G$ inherits the assigned angles from $G$ in the sense that to every vertex $p=\pi(z, i)$ of an edge $E_{i}$ in $H$ we assign $d^{z, i} \in \mathbb{S}^{d-1}$ coinciding with the assignment of $G$.

For the convenience of the reader, let us recall here the key notion of stratified straight subgraph, which was already presented in Definition 1.3.

Definition 3.6 (Stratified straight subgraph) Given a graph $G$ with assigned angles we say that a subgraph $H \subseteq G$ is stratified-straight if there exists a finite sequence of subgraphs, called strata,

$$
\emptyset=H_{q} \subset H_{q-1} \subset \ldots \subset H_{1} \subset H_{0}=H
$$

and maps $\Sigma_{j}: H_{j} \rightarrow \mathbb{R}^{d}$ such that for $j=0, \ldots, q-1$ we have that

- the (sub)network $\left(H_{j}, \Sigma_{j}\right)$ satisfies the angle condition in the sense of Definition 3.2 with (real or virtual) tangent vectors coinciding with the ones associated to $\left(H_{0}, \Sigma_{0}\right)$ and whose regular curves are straight segments;

- $H_{j+1}=\operatorname{Sing}\left(\left(H_{j}, \Sigma_{j}\right)\right)$.

We call step of $G$ the least $q$ for which the above holds.

Every straight graph with assigned angles is trivially stratified-straight, but the converse does not hold in general as shown by the following example.

Example 3.7 We consider the graph of Fig. 5 characterized by the following identifications:

$$
(0,1) \sim(0,2),(1,2) \sim(1,3) \sim(0,5),(1,1) \sim(0,3) \sim(0,4),(1,4) \sim(1,5),
$$


$\measuredangle d^{0,2}, d^{0,1}=\measuredangle d^{0,3}, d^{0,4}=0, \quad \measuredangle d^{1,1}, d^{0,3}=\measuredangle d^{1,3}, d^{1,2}=\measuredangle d^{1,4}, d^{1,5}=\frac{\pi}{2}$,

where $\measuredangle v, w \in[0,2 \pi)$ here identifies the least positive angle between two vectors $v, w$ in the plane (see Fig. 5 on the left).

It is clearly not possible to construct a triangle with strictly positive length of its three (straight) edges with angles $0, \frac{\pi}{2}, \frac{\pi}{2}$. The minimal step of $G$ seen as a stratified straight graph is then 2, with $H_{0}=G, H_{1}=E_{3} \cup E_{4} \cup E_{5}$ and $H_{2}=E_{5}$.

Notice that the notion of straight and stratified straight graphs coincide when the underlying graph $G$ has the structure of a tree with no cycles. In Sect. 7 we introduce a class of graphs possibly with cycles for which every stratified straight subgraph is also straight.

Definition 3.8 (Degenerate $N$-network) Let $G$ be an $N$-graph with assigned angles. A network $\mathcal{N}=(G, \Gamma)$ is degenerate if

- it satisfies the angle condition in the sense of Definition 3.2, with (real or virtual) tangents $\tau^{z, i}$;

- its singular part $\operatorname{Sing}(\mathcal{N})$ is a stratified-straight subgraph with (real or virtual) tangents coinciding with the $\tau^{z, i}$ above.

We denote by $\mathcal{C}_{\text {Deg }}$ the class of degenerate networks. We remark that by definition $\mathcal{C}_{\text {Reg }} \subset \mathcal{C}_{\text {Deg. }}$. We remind that to compute the elastic energy of a network in $\mathcal{C}_{\text {Deg }}$ we make use of the extension of the functional defined in (2.3).

Proposition 3.9 (Compactness) Let $\left\{\mathcal{N}_{n}\right\}_{n \in \mathbb{N}}$ be a sequence of networks in $\mathcal{C}_{\text {Deg }}$ such that

$$
\limsup _{n} \widetilde{\mathcal{E}}\left(\mathcal{N}_{n}\right) \leq C<+\infty
$$

Then $\mathcal{N}_{n}$ converges (up to subsequence and translation) to $\mathcal{N}_{\infty} \in \mathcal{C}_{\text {Deg }}$ weakly in $H^{2}$ and strongly in $C^{1, \alpha}$ for every $\alpha \in(0,1 / 2)$. In particular if $\left\{\mathcal{N}_{n}\right\}_{n \in \mathbb{N}}$ is a sequence of networks in $\mathcal{C}_{\text {Reg }}$, then $\mathcal{N}_{n} \stackrel{H^{2}}{\rightarrow} \mathcal{N}_{\infty} \in \mathcal{C}_{\text {Deg. }}$.

Proof Up to subsequence and up to relabeling the edges $E_{i}$ we can suppose that for a certain $k \in\{1, \ldots N\}$ for every $n \in \mathbb{N}$ the curves $\mathcal{N}_{n}^{1}, \ldots, \mathcal{N}_{n}^{k}$ are regular and the curves $\mathcal{N}_{n}^{k+1}, \ldots, \mathcal{N}_{n}^{N}$ are singular. Without loss of generality we suppose that for every $n \in \mathbb{N}$ and for every $i \in\{1, \ldots, k\}$ the immersions $\gamma_{n}^{i}:=\left.\Gamma_{n}\right|_{E_{i}}:[0,1] \rightarrow \mathbb{R}^{d}$ are regular parametrizations with constant speed equal to the length and we ask that $\gamma_{n}^{1}(0)$ is mapped into the origin of $\mathbb{R}^{d}$. For every $i \in\{1, \ldots, k\}$ and for every $n \in \mathbb{N}$ we have that

$$
\begin{aligned}
& \left\|\gamma_{n}^{i}\right\|_{\infty}=\sup _{x \in[0,1]}\left|\gamma_{n}^{i}(x)\right| \leq L\left(\mathcal{N}_{n}\right) \leq \widetilde{\mathcal{E}}\left(\mathcal{N}_{n}\right) \leq C<\infty, \\
& \left\|\dot{\gamma}_{n}^{i}\right\|_{\infty}=\sup _{x \in[0,1]}\left|\dot{\gamma}_{n}^{i}(x)\right|=\ell\left(\mathcal{N}_{n}^{i}\right) \leq L\left(\mathcal{N}_{n}\right) \leq \widetilde{\mathcal{E}}\left(\mathcal{N}_{n}\right) \leq C<\infty,
\end{aligned}
$$



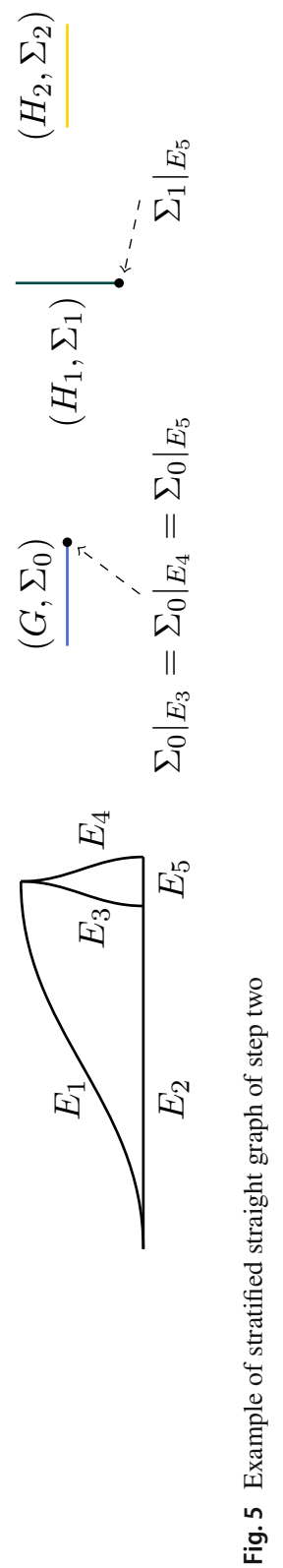


$$
\left\|\ddot{\gamma}_{n}^{i}\right\|_{2}^{2}=\ell\left(\mathcal{N}_{n}^{i}\right)^{3} \int_{0}^{1} \frac{\left|\ddot{\gamma}_{n}^{i}(x)\right|^{2}}{\ell\left(\mathcal{N}_{n}^{i}\right)^{3}} \mathrm{~d} x \leq L\left(\mathcal{N}_{n}\right)^{3} \widetilde{\mathcal{E}}\left(\mathcal{N}_{n}\right) \leq C^{4}<\infty .
$$

Since for every $i \in\{k+1, \ldots, N\}$ the maps are constant on the bounded interval of parametrization $[0,1]$, a bound on $\|\cdot\|_{H^{2}}$ is trivially obtained. Then for every $i \in\{1, \ldots, N\}$ up to a subsequence (not relabeled) $\gamma_{n}^{i} \rightarrow \gamma_{\infty}^{i}$ weakly in $H^{2}(0,1)$ and thanks to classical compact embedding theorems $\gamma_{n}^{i} \rightarrow \gamma_{\infty}^{i}$ strongly in $C^{1, \alpha}([0,1])$ for every $\alpha \in\left(0, \frac{1}{2}\right)$.

Since all the networks of the sequence $\left\{\mathcal{N}_{n}\right\}_{n \in \mathbb{N}}$ satisfy the angle condition of Definition 3.2, for each $n$ there exists a family of unit vectors $\mathcal{T}_{n}=\left(\tau_{n}^{z, i}\right)_{(z, i) \in \pi^{-1}\left(V_{G}\right)}$ that are either the real or the virtual tangents. By compactness of $\mathbb{S}^{d-1}$ up to subsequence (not relabeled) $\mathcal{T}_{n}$ converge to a limit $\mathcal{T}_{\infty}$ composed of elements denoted by $\tau_{\infty}^{z, i}$. Notice that for the the indices $i$ for which $\ell\left(\gamma_{\infty}^{i}\right)>0$ we have that (up to subsequence) $(-1)^{z} \frac{\dot{\gamma}_{n}^{i}}{\left|\dot{\gamma}_{n}^{i}\right|}$ converge to $\tau_{\infty}^{z, i}$.

Define $\Gamma_{\infty}: G \rightarrow \mathbb{R}^{2}$ in such a way that $\left.\Gamma_{\infty}\right|_{E_{i}}=\gamma_{\infty}^{i}$ and call $\mathcal{N}_{\infty}:=\left(G, \Gamma_{\infty}\right)$. We want to prove that $\mathcal{N}_{\infty} \in \mathcal{C}_{\text {Deg. }}$.

First of all we claim that the angle condition is verified for $\mathcal{N}_{\infty}$ with the family $\mathcal{T}_{\infty}$. It is easy to check that there exist rotations $\mathrm{R}_{p}$ (obtained as limits of those for $\mathcal{N}_{n}$ ) that verify (3.1). It remains to prove that for the regular curves $\gamma_{\infty}^{i}$ the vectors $\tau_{\infty}^{i}$ coincide with the outer tangents and that for the constant curves we have $\tau_{\infty}^{0, i}=-\tau_{\infty}^{1, i}$. For each $i \in\{1, \ldots, N\}$ there are three possible cases: a regular curve converges to a regular curve; a regular curve converges to a constant curve; a constant curve converges to a constant curve. In the first case the claim follows from the above argument. In the second case the claim follows by Lemma 3.1. In the third case the claim follows trivially.

It remains to verify that $H=\operatorname{Sing}\left(\mathcal{N}_{\infty}\right)$ is stratified-straight. We proceed by induction: we set $H_{0}=H$ and supposing to have obtained $H_{i}$ we construct $\Sigma_{i}$ and $H_{i+1}$. We can assume without loss of generality that $H_{i}$ is connected (otherwise we apply the same argument to each connected component) and up to a translation that $0 \in \Gamma_{n}\left(H_{i}\right)$. We define $L_{n}^{i}:=L\left(\Gamma_{n}\left(H_{i}\right)\right)$ and consider the rescaled networks $\mathcal{N}_{n}^{(i)}=\left(H_{i}, \Gamma_{n}^{(i)}\right)$ with

$$
\Gamma_{n}^{(i)}=\frac{1}{L_{n}^{i}} \Gamma_{n}
$$

Then $L\left(\mathcal{N}_{n}^{(i)}\right)=1$. Moreover, at each step the network $\mathcal{N}_{n}^{(i)}$ is defined starting from the singular part of the previous one (indeed $H_{0}=H$, while for $H_{i}, i \geq 1$, see (3.6) below), therefore we have that $L_{n}^{i} \rightarrow 0$. Thanks to the scaling property (2.5) we thus have

$$
\int_{\mathcal{N}_{n}^{(i)}}\left|\vec{k}^{i}\right|^{2} \mathrm{~d} s=L_{n}^{i} \int_{\Gamma_{n}\left(H_{i}\right)}\left|\vec{k}^{i}\right|^{2} \mathrm{~d} s \rightarrow 0 \text { as } n \rightarrow \infty
$$


and in particular $\widetilde{\mathcal{E}}\left(\mathcal{N}_{n}^{(i)}\right) \leq C<\infty$ for some constant $C$. Hence we can repeat the previous reasoning to conclude that up to subsequence the networks $\mathcal{N}_{n}^{(i)}$ converge weakly in $H^{2}$ and strongly in $C^{1}$ to a network $\mathcal{N}_{\infty}^{(i)}=\left(H_{i}, \Gamma_{\infty}^{(i)}\right)$. By $C^{1}$-convergence $L\left(\mathcal{N}_{\infty}^{(i)}\right)=1$ and thus the limit network has at least one regular curve. By Lemma 3.1 we have that the curves of $\mathcal{N}_{\infty}^{(i)}$ are either constant or straight segments, since all surviving curves have a constant tangent. We then define

$$
\Sigma_{i}=\Gamma_{\infty}^{(i)} \quad H_{i+1}=\operatorname{Sing}\left(\left(H_{i}, \Sigma_{i}\right)\right)
$$

and we repeat the process until $\operatorname{Sing}\left(\left(H_{i}, \Sigma_{i}\right)\right)=\emptyset$.

After a finite number of steps the process stops and we obtain that the strata $\emptyset=$ $H_{q} \subset \ldots \subset H_{0}=H$ by construction satisfy the condition of Definition 3.6.

We have thus concluded that $\mathcal{N}_{\infty}$ satisfies the angle condition with (real or virtual) tangents $\mathcal{T}_{\infty}$ and $\operatorname{Sing}\left(\mathcal{N}_{\infty}\right)$ is stratified-straight whose (real or virtual) tangents are consistent with $\mathcal{T}_{\infty}$, and thus by Definition $3.8 \mathcal{N}_{\infty} \in \mathcal{C}_{\text {Deg }}$.

To convince the reader that in Definition 3.8 we cannot replace the notion of stratified straight subgraph by the simpler one of straight subgraphs, and hence the definition of degenerate networks is in a sense sharp, we give an example of a sequence of regular networks with equibounded energy which converges to a singular network whose singular part is stratified straight but nor straight.

Example 3.10 Consider a 5-graph $G$ where in particular

$$
\begin{aligned}
& (0,1) \sim(0,2), \quad(1,1) \sim(0,3),(1,2) \sim(1,3), \\
& \measuredangle d^{0,1}, d^{0,2}=0, \quad \measuredangle d^{1,1}, d^{0,3}=\measuredangle d^{1,2}, d^{1,3}=\frac{\pi}{2},
\end{aligned}
$$

where $\measuredangle v, w \in[0,2 \pi)$ here identifies the least positive angle between two vectors $v, w$ in the plane (see Fig. 6 on the left).

We construct a sequence of regular networks $\mathcal{N}_{n}=\left(G, \Gamma_{n}\right)$ that converge as $n \rightarrow \infty$ to a degenerate network whose singular part is stratified straight.

Consider three immersions $\gamma^{1}, \gamma^{2}, \gamma^{3}:[0,1] \rightarrow \mathbb{R}^{2}$ such that the curve $\gamma^{3}([0,1])$ is an arc of circle of radius $r$ and length $2 \alpha r$ and both $\gamma^{1}([0,1])$ and $\gamma^{2}([0,1])$ are arcs of circle of radius $R$ and length $\alpha R$ with $R=\frac{\sin \left(\frac{\pi}{2}-\alpha\right)}{\sin \alpha} r$. Then

$$
\mathcal{E}\left(\gamma^{1}\right)+\mathcal{E}\left(\gamma^{2}\right)+\mathcal{E}\left(\gamma^{3}\right)=2\left(r \alpha \cot \alpha+\frac{\alpha \tan \alpha}{r}\right)+2 \alpha r+\frac{2 \alpha}{r} .
$$

We construct a sequence of immersions $\gamma_{n}^{1}, \gamma_{n}^{2}, \gamma_{n}^{3}$ simply by taking $r_{n}=\frac{1}{n}$ and $\alpha_{n}=\frac{1}{n^{2}}$. Then as $n \rightarrow \infty$ the lengths of the three curves go to zero and

$$
\mathcal{E}\left(\gamma_{n}^{1}\right)+\mathcal{E}\left(\gamma_{n}^{2}\right)+\mathcal{E}\left(\gamma_{n}^{3}\right)=2\left(\frac{1}{n}+\frac{1}{n^{3}}\right)+\frac{2}{n^{3}}+\frac{2}{n}+o\left(\frac{1}{n^{5}}\right) \stackrel{n \rightarrow \infty}{\longrightarrow} 0 .
$$

Moreover it is not possible to construct a triangle with three edges of positive length and angles $0, \frac{\pi}{2}, \frac{\pi}{2}$. 

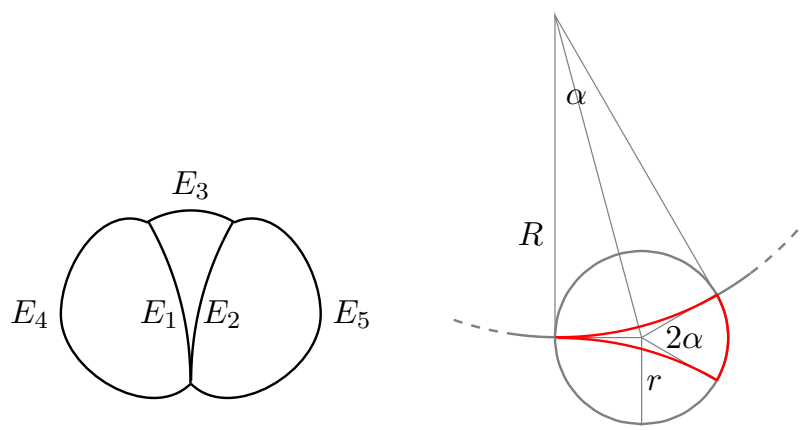

Fig. 6 Representation of the graph $G$ with assigned angles and construction of the sequence

Notice that the energy of the sequence need not be infinitesimal. In fact by taking $r_{n}=\alpha_{n}=\frac{1}{n}$, one gets that the above energy converges to 2 . This highlights the fact that curves of a network need not become flatter and flatter along a sequence even if their length goes to zero. Hence our characterization of the class of degenerate networks is not related to the curvature of the approximating sequences.

\section{Relaxation}

Let us define the following extended problem.

Problem 4.1 Given an $N$-graph $G$ with assigned angles we want to find

$$
\inf \{\overline{\mathcal{E}}(\mathcal{N}) \mid \mathcal{N}=(G, \Gamma) \text { is a (possibly) singular network }\}
$$

where $\overline{\mathcal{E}}$ is the extension of $\mathcal{E}$ defined as

$$
\overline{\mathcal{E}}(\mathcal{N}):= \begin{cases}\widetilde{\mathcal{E}}(\mathcal{N}) & \text { if } \mathcal{N} \in \mathcal{C}_{\text {Deg }} \\ +\infty & \text { otherwise }\end{cases}
$$

The reason to consider this functional will be clear at the end of this section: we will prove that it coincides with the relaxation of the functional $\mathcal{E}$ defined in (2.2), and thus it is the natural extension of $\mathcal{E}$ from $\mathcal{C}_{\text {Reg }}$ to $\mathcal{C}_{\text {Deg }}$.

Proposition 4.2 (Recovery sequence) For every network $\mathcal{N}=(G, \Gamma) \in \mathcal{C}_{\text {Deg }}$ there exists a sequence of networks $\left\{\mathcal{N}_{n}\right\}_{n \in \mathbb{N}}$ such that

$$
\mathcal{N}_{n} \stackrel{H^{2}}{\longrightarrow} \mathcal{N} \text { and } \mathcal{E}\left(\mathcal{N}_{n}\right) \rightarrow \overline{\mathcal{E}}(\mathcal{N})
$$

To prove the above proposition we will take advantage of the following preliminary lemmas.

We recall the following version of a classical lemma. 
Lemma 4.3 Let $f \in L^{2}\left([0,1] ; \mathbb{R}^{d}\right)$. Then

$$
\|f-f \circ \phi\|_{L^{2}} \rightarrow 0 \quad \text { as }\|\phi-I d\|_{C^{0}(0,1)} \rightarrow 0
$$

where $\phi$ varies among all continuous maps $\phi:[0,1] \rightarrow[0,1]$.

Proof By the triangle inequality and the density of continuous functions on $L^{2}$ it is sufficient to prove the result when $f$ is continuous. In this case, being defined on a compact set, $f$ is uniformly continuous with some modulus of continuity $\omega_{f}$. Then

$$
\int_{0}^{1}|f(t)-f(\phi(t))|^{2} \mathrm{~d} t \leq \omega_{f}\left(\|\phi-I d\|_{C^{0}}\right)^{2} \rightarrow 0 \quad \text { as } \quad\|\phi-I d\|_{C^{0}} \rightarrow 0 .
$$

Lemma 4.4 Let $l \in(0,+\infty)$ and $0 \leq \delta \leq l$. Let $\gamma:[0,1] \rightarrow \mathbb{R}^{d}$ and $\sigma:\left[1,1+\frac{\delta}{l}\right] \rightarrow$ $\mathbb{R}^{d}$ be two curves of class $H^{2}$ with $\gamma(1)=\sigma(1), \dot{\gamma}(1)=\dot{\sigma}(1)$ and with constant speed $|\dot{\gamma}| \equiv|\dot{\sigma}| \equiv$ l. Consider the concatenation $\gamma * \sigma:\left[0,1+\frac{\delta}{l}\right] \rightarrow \mathbb{R}^{d}$ defined in the natural way and its linear reparametrization $\gamma_{\sigma}:[0,1] \rightarrow \mathbb{R}^{d}$ given by

$$
\gamma_{\sigma}(t)=(\gamma * \sigma)\left(\left(1+\frac{\delta}{l}\right) t\right)
$$

Then

$$
\left\|\gamma-\gamma_{\sigma}\right\|_{H^{2}(0,1)} \leq \eta(\mathcal{E}(\sigma))
$$

where $\eta$ is a function such that $\eta(z) \rightarrow 0$ as $z \rightarrow 0^{+}$.

Proof It is sufficient to prove the inequality for the second derivatives, because then by the Poincaré inequality we obtain the inequality for the full $H^{2}$ norm. We have that:

$$
\begin{aligned}
\int_{0}^{1}\left|\ddot{\gamma}(t)-\ddot{\gamma}_{\sigma}(t)\right|^{2} \mathrm{~d} t & =\int_{0}^{\frac{1}{1+\frac{\delta}{l}}}\left|\ddot{\gamma}(t)-\ddot{\gamma}\left(\left(1+\frac{\delta}{l}\right) t\right)\right|^{2} \mathrm{~d} t+\int_{\frac{1}{1+\frac{\delta}{l}}}^{1}\left|\ddot{\gamma}(t)-\ddot{\sigma}\left(\left(1+\frac{\delta}{l}\right) t\right)\right|^{2} \mathrm{~d} t \\
& \leq \int_{0}^{\frac{1}{1+\frac{\delta}{l}}}\left|\ddot{\gamma}(t)-\ddot{\gamma}\left(\left(1+\frac{\delta}{l}\right) t\right)\right|^{2} \mathrm{~d} t+2 \int_{\frac{1}{1+\frac{\delta}{l}}}^{1}|\ddot{\gamma}(t)|^{2} \mathrm{~d} t \\
& +2 \int_{\frac{1}{1+\frac{\delta}{l}}}^{1}\left|\ddot{\sigma}\left(\left(1+\frac{\delta}{l}\right) t\right)\right|^{2} \mathrm{~d} t .
\end{aligned}
$$

Thanks to Lemma 4.3 the first term of the last expression goes to zero as $\delta \rightarrow 0$. The second term goes to zero as $\delta \rightarrow 0$ by the absolute integrability of $|\ddot{\gamma}|^{2}$. Since $\delta=\ell(\sigma)$, then the first two terms go to zero as $\mathcal{E}(\sigma) \rightarrow 0$. After the change of variables $x=\left(1+\frac{\delta}{l}\right) t$, the third term reads

$$
\frac{2}{1+\frac{\delta}{l}} \int_{1}^{1+\frac{\delta}{l}}|\ddot{\sigma}(x)|^{2} \mathrm{~d} x
$$


Since $\sigma$ is parametrized with constant speed $l$, recalling (2.1) we have

$$
\int_{1}^{1+\frac{\delta}{l}}|\ddot{\sigma}(x)|^{2} \mathrm{~d} x=l^{3} \int_{\sigma}\left|\vec{k}_{\sigma}\right|^{2} \mathrm{~d} s \leq l^{3} \mathcal{E}(\sigma) .
$$

Therefore all three terms go to zero as $\mathcal{E}(\sigma) \rightarrow 0$, and the thesis is proven.

Remark 4.5 We will use the previous lemma in the following way: whenever we have a curve $\gamma$ parametrized with constant speed and we concatenate it with another curve $\sigma$, then the constant speed parametrization on $[0,1]$ of $\gamma * \sigma$ is close in $H^{2}$ to $\gamma$ if $\mathcal{E}(\sigma)$ is small.

Lemma 4.6 (Change of train tracks) Consider two parallel horizontal straight lines in $\mathbb{R}^{2}$ at distance $h$. There exists an embedded curve located between the two lines such that it connects the two lines spanning a horizontal interval of length $b \approx 2 \sqrt{h}$ and with energy $\approx 4 \sqrt{h}$, asymptotically as $h \rightarrow 0$. More precisely, there exists a regular curve $\gamma:[0,1] \rightarrow \mathbb{R}^{2}$ such that

$$
\frac{\dot{\gamma}(0)}{|\dot{\gamma}(0)|}=\frac{\dot{\gamma}(1)}{|\dot{\gamma}(1)|}=e_{1}, \quad \gamma(0)=(0,0), \quad \gamma(1)=(b, h),
$$

with

$$
b=2 \sqrt{h}(1+o(1)), \text { and } \mathcal{E}(\gamma)=4 \sqrt{h}(1+o(1)) .
$$

Proof We construct the curve by putting together two congruent arcs of circle of radius 1 as in Fig. 7. The modulus of the curvature is 1 and the angle is $\theta$. The total length is $2 \theta$ which also equals the total squared curvature of the arcs. The total energy $\mathcal{E}(\gamma)$ is thus $4 \theta$. The height $h$ and the base $b$ are related to $\theta$ by

$$
h=2(1-\cos \theta) \approx \theta^{2} \quad b=2 \sin \theta \approx 2 \theta \text { as } \theta \rightarrow 0,
$$

and therefore given $h$ we can choose $\theta=\arccos \left(1-\frac{h}{2}\right) \approx \sqrt{h}$ to obtain

$$
b \approx 2 \sqrt{h} \quad \mathcal{E}(\gamma) \approx 4 \sqrt{h}
$$

The following lemma allows us to suppose, in the construction of the recovery sequence, that each regular curve of $\mathcal{N}$ is straight near its endpoints, up to a small perturbation and a small change of the value of its elastic energy.

Lemma 4.7 (Straightening at vertices) Consider a curve $\gamma:[0,1] \rightarrow \mathbb{R}^{d}$ of class $H^{2}$ parametrized with constant speed. Then for every $\varepsilon>0$ there exists a curve $\tilde{\gamma}:[0,1] \rightarrow \mathbb{R}^{d}$ of class $H^{2}$ parametrized with constant speed such that on some 
Fig. 7 The construction of Lemma 4.6

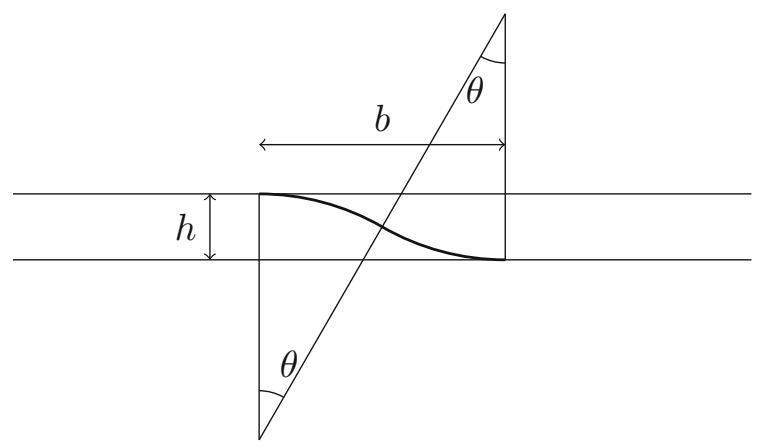

interval $[0, a]$ its image is a straight segment of length at most $\varepsilon$ with direction $\gamma^{\prime}(0)$, and such that

$$
\gamma(0)=\tilde{\gamma}(0), \quad\|\gamma-\tilde{\gamma}\|_{H^{2}} \leq \varepsilon, \quad \mathcal{E}(\tilde{\gamma}) \leq \mathcal{E}(\gamma)(1+\varepsilon)
$$

Proof Without loss of generality we suppose that $\gamma(0)=0, \dot{\gamma}(0)=e_{1}$, and for $\delta>0$ we define

$$
\gamma_{\delta}(t)=\gamma(t)+\delta \psi(t) e_{1}
$$

where $\psi:[0,1] \rightarrow \mathbb{R}$ is a fixed $C^{2}$ map with

$$
\psi(t)=1 \text { for } t \in\left[0, \frac{1}{4}\right] \quad \text { and } \quad \psi(t)=0 \text { for } t \in\left[\frac{1}{2}, 1\right] .
$$

Then $\left\|\gamma-\gamma_{\delta}\right\|_{H^{2}}=\delta\|\psi\|_{H^{2}}$, and as a consequence $\mathcal{E}\left(\gamma_{\delta}\right) \leq \mathcal{E}(\gamma)(1+C \delta)$ for some constant $C$ that depends on $\|\psi\|_{H^{2}}$. Let $\alpha_{\delta}$ be the constant speed parametrization of the concatenation of a straight segment from 0 to $\delta e_{1}$ and $\gamma_{\delta}$. From Lemma 4.4 it follows that $\left\|\alpha_{\delta}-\gamma_{\delta}\right\|_{H^{2}(0,1)}$ tends to zero as $\delta \rightarrow 0$. Hence the thesis follows by taking $\tilde{\gamma}=\alpha_{\delta}$ for $\delta>0$ sufficiently small.

We now come to the proof of the recovery sequence.

Proof of Proposition 4.2 Consider $\mathcal{N}=(G, \Gamma) \in \mathcal{C}_{\text {Deg }}$ and let $H=H_{0}=\operatorname{Sing}(\mathcal{N})$. Without loss of generality we can assume that the regular curves of $\mathcal{N}$ are parametrized with constant speed equal to their length. For simplicity we can suppose that $H$ is connected and $\Gamma(H)$ is the origin of $\mathbb{R}^{d}$. In the case of many connected components it is sufficient to repeat the argument for each one of them. By assumption there exist (real or virtual) tangents $\mathcal{T}_{G}=\left(\tau^{z, i}\right)_{(z, i) \in \pi^{-1}\left(V_{G}\right)}$ of the graph $G$, such that $H$ is stratified-straight with strata

$$
\emptyset=H_{q} \subset H_{q-1} \subset \ldots \subset H_{1} \subset H_{0}=H
$$

with $\mathbb{N} \ni q \leq N$, and such that the (real or virtual) tangents of $\left(H_{i}, \Sigma_{i}\right)$ coincide with the corresponding ones of $\mathcal{T}_{G}$. 
Fix $\varepsilon>0$. Consider the family $\mathcal{F}$ of regular curves of $\mathcal{N}$ that have an endpoint in $H$. For every $\gamma^{i} \in \mathcal{F}$ we have that $\Gamma(\pi(z, i))=0$ whenever $p=\pi(z, i) \in H$ and moreover by Lemma 4.7 we can suppose that near $p$ the image of the curve $\gamma^{i}$ coincides with a straight segment of length $l \leq \varepsilon$, up to adding a small error of order at most $\varepsilon$ to the energy.

We consider the first stratum $H=H_{0}$ and up to rescaling we can suppose that $\Sigma_{0}\left(H_{0}\right) \subset B_{l^{3}}(0)$ where we recall that $0=\Gamma\left(H_{0}\right)$. We want to partially desingularize $H$, that is we replace in a neighborhood of 0 the completely singular subnetwork $\left(H_{0}, \Gamma\right)$ with the first stratum $\left(H_{0}, \Sigma_{0}\right)$. We have to make sure to connect each curve to the right junction and with the right angle.

Consider a curve $\gamma^{i} \in \mathcal{F}$, and suppose for instance that $p=\pi((0, i)) \in V_{H}$ (the case when $\pi((1, i)) \in V_{H}$ is completely analogous). Then there exists $a^{0, i}$ such that $\left.\gamma^{i}\right|_{\left[0, a^{0, i}\right]}$ is a straight segment of length $l$. In particular $\dot{\gamma}^{i}\left(a^{0, i}\right)$ is parallel to $\dot{\gamma}^{i}(0)=\tau^{0, i}$. We now consider the parallel lines with direction $\tau^{0, i}$ passing through the point $\Sigma_{0}((0, i))$ and through the point $\gamma^{i}\left(a^{0, i}\right)$. Their distance is of order $l^{3}$. We can thus apply Lemma 4.6 to modify the curve $\gamma^{i}$ on the interval $\left[0, a^{0, i}\right]$ in order to connect $\Sigma_{0}((0, i))$ to $\gamma^{i}\left(a^{0, i}\right)$ with an energy of order $l^{3 / 2} \leq \varepsilon^{3 / 2}$ and with outer tangent $\tau^{0, i}$. Finally we reparametrize the modified curve $\gamma^{i}$ on $[0,1]$ by constant speed equal to its length. By Remark 4.5 it follows that the modified curve $\gamma^{i}$ is also close to the original one in $H^{2}$-norm.

We repeat this process for every curve in $\mathcal{F}$ and every endpoint in $V_{H}$. By construction the angle condition is still verified for the newly constructed network, but now the stratum $H_{0}$ is not completely singular anymore. We then repeat the same process for each stratum until we obtain a regular network $\mathcal{N}_{\varepsilon}$ whose energy is at most $\overline{\mathcal{E}}(\mathcal{N})+C \varepsilon$ and whose $H^{2}$ distance from $\mathcal{N}$ is at most $C \varepsilon$.

Since we can do this for any $\varepsilon>0$, choosing $\varepsilon_{n}=\frac{1}{n}$ we obtain a family $\mathcal{N}_{n}$ of regular networks that approximate $\mathcal{N}$ and thus satisfies the thesis of the theorem.

Theorem 4.8 The functional $\overline{\mathcal{E}}$ is the lower semicontinuous envelope of the functional $\mathcal{E}$ with respect to the weak convergence in $H^{2}$, that is

$$
\overline{\mathcal{E}}\left(\mathcal{N}_{\infty}\right)=\inf \left\{\liminf _{n \rightarrow \infty} \mathcal{E}\left(\mathcal{N}_{n}\right) \mid \mathcal{N}_{n} \in \mathcal{C}_{\text {Reg }}, \mathcal{N}_{n} \stackrel{H^{2}}{\rightarrow} \mathcal{N}_{\infty}\right\}
$$

\section{Moreover the relaxed functional $\overline{\mathcal{E}}$ admits a minimizer.}

Proof The existence of minimizers of the functional $\overline{\mathcal{E}}$ follows by a direct method in the Calculus of Variations combining the lower semicontinuity of $\overline{\mathcal{E}}$ and the compactness, that we both gain thanks to Propositions 3.9 and 4.2.

By definition $\overline{\mathcal{E}} \leq \mathcal{E}$ in the class $\mathcal{C}_{\text {Reg }}$. Let $\mathcal{F}$ be a lower semincontinuous functional defined in the class of $N$-networks such that $\mathcal{F} \leq \mathcal{E}$ in the class $\mathcal{C}_{\text {Reg }}$. Proposition 4.2 implies that $\overline{\mathcal{E}}(\mathcal{N}) \geq \mathcal{F}(\mathcal{N})$ in the class of $N$-networks and this concludes also the first part of the statement.

Remark 4.9 The choice of the weak topology in the above relaxation result is the natural one because of the compactness result of Proposition 3.9. However we observe that, as 
Fig. 8 A representation of the graph $G$ of a Theta-network

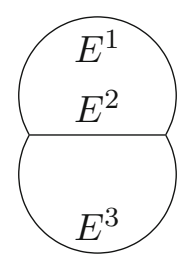

is clear from Proposition 4.2, a slightly finer result holds: while the liminf inequality $\overline{\mathcal{E}}\left(\mathcal{N}_{\infty}\right) \leq \liminf _{n \rightarrow \infty} \mathcal{E}\left(\mathcal{N}_{n}\right)$ holds for every sequence $\mathcal{N}_{n}$ converging weakly to $\mathcal{N}_{\infty}$, the limsup inequality holds with the strong topology, namely, for every $\mathcal{N}_{\infty}$ there exists a sequence $\mathcal{N}_{n}$ converging strongly to $\mathcal{N}_{\infty}$ such that $\overline{\mathcal{E}}\left(\mathcal{N}_{\infty}\right) \geq \lim \sup _{n \rightarrow \infty} \mathcal{E}\left(\mathcal{N}_{n}\right)$ (and therefore equality holds for the limit). Recalling that the lower semicontinuous envelope of a functional $F$ coincides with the $\Gamma$-limit of the constant sequence $F_{n}=F$, we can here make a comparison with the difference between Mosco convergence and $\Gamma$-convergence.

\section{Lower Bounds on the Energy}

Now that we have shown that the relaxed Problem 4.1 admits a minimizer, one may wonder if in some cases the minimizer is regular, that is if Problem 2.13 has a minimum. We report here on a special case in which we get the desired result.

Definition 5.1 We call Theta-network any planar network $(G, \Gamma)$ where $G$ is a 3-graph composed of edges $E_{1}, E_{2}, E_{3}$ with the identifications

$$
(0,1) \sim(0,2) \sim(0,3) \text { and }(1,1) \sim(1,2) \sim(1,3)
$$

with assigned directions $\left\{d^{0, i}\right\}_{i=1}^{3},\left\{d^{1, i}\right\}_{i=1}^{3}$ such that at any junction the assigned vectors form three (equal) angles equal to $\frac{2}{3} \pi$.

The minimization of the elastic energy among Theta-networks has been considered in [8]. It turns out that in this class the minimizers of the relaxed problem are regular networks (see [8, Theorem 4.10]) and hence Problem 2.13 admits a minimizer.

Although we have proven that in general sequences of regular networks with equibounded energy converge to a limit network in $\mathcal{C}_{\text {Deg }}$ one may think that for every choice of the topology of the graph $G$ and of the angle condition minimizers are actually in $\mathcal{C}_{\text {Reg. }}$. The following example shows that even for very simple topologies of $G$ this could not be the case and so it is hopeless to give always a positive answer to Problem 2.13.

Example 5.2 (The minimizers of the relaxed problem are degenerate) Consider the 3-graph $G$ with assigned angles composed of $E_{1}, E_{2}, E_{3}$ with $\pi(0,1)=\pi(1,1)=$ $\pi(0,2)$ and $\pi(1,2)=\pi(0,3)=\pi(1,3)$ depicted in Fig. 9. Then the length of one of the curves of the minimizers is zero. 
Fig. 9 On the left a representation of the graph $G$. On the right a possible minimizer $\mathcal{N}_{\min }=\left(G, \Gamma_{\min }\right)$
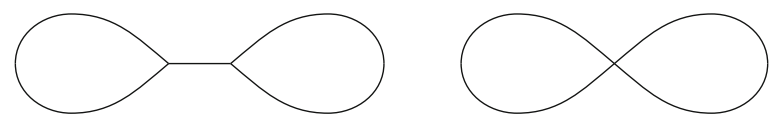

We have just seen in Example 5.2 that in some situations the minimizers of Problem 2.13 are degenerate networks. We want to understand under which conditions minimizers have at least some curves with positive length and do not degenerate to a point. To be more precise, consider a graph $G$ with assigned angles. We recall that $\mathcal{D}$ is the set of the assigned directions $d^{i, z}$ (see Definition 2.10). We define the minimization problem

$$
I_{\mathcal{D}}:=\min \{\overline{\mathcal{E}}(\mathcal{N}) \mid \mathcal{N}=(G, \Gamma) \text { is a network }\}
$$

We are interested in conditions on the set $\mathcal{D}$ that guarantee that $I_{\mathcal{D}}$ is strictly positive.

Lemma 5.3 Let $\gamma:[0, \ell(\gamma)] \rightarrow \mathbb{R}^{2}$ be a regular, continuous, piecewise $H^{2}$ curve parametrized by arclength such that $\gamma(0)=0, \dot{\gamma}(0)=(\cos \theta, \sin \theta)$ for some $\theta \in$ $(-\pi, \pi]$, and $\dot{\gamma}(\ell(\gamma))=(1,0)$. Then

$$
\int_{\gamma}|\vec{k}| \mathrm{d} s \geq|\theta|
$$

and the equality holds if $\gamma$ is convex, i.e., if $\langle\vec{k}, v\rangle$ remains non negative (or non positive) along the curve, where $v$ is the counterclockwise rotation of $\frac{\pi}{2}$ of the tangent vector $\tau$ along $\gamma$.

Moreover suppose that for every $i \in\{1, \ldots, N\}$ the curve $\gamma^{i}$ is regular, continuous and of class $H^{2}$ and suppose that there exists $\alpha>0$ such that

$$
\sum_{i=1}^{N} \int_{\gamma^{i}}\left|\vec{k}^{i}\right| \mathrm{d} s \geq \alpha
$$

then $\mathcal{E}\left(\cup_{i=1}^{N} \gamma^{i}\right) \geq 2 \alpha$.

Proof Denote by $\tau$ the unit tangent vector to the curve $\gamma$. Since the curve $\gamma$ is parametrized by arclength, we have that $\dot{\gamma}=\tau$ and $\ddot{\gamma}=\dot{\tau}=\vec{k}$. By hypothesis the angle spanned by the unit tangent vector and the horizontal axis is greater or equal than $|\theta|$, hence

$$
\int_{\gamma}|\vec{k}(s)| \mathrm{d} s=\int_{0}^{\ell(\gamma)}|\dot{\tau}(s)| \mathrm{d} s \geq|\theta| .
$$

By writing $\tau=(\cos \varphi(s), \sin \varphi(s))$ for some function $\varphi(s) \in(-\pi, \pi]$, as $|\dot{\tau}|=|\dot{\varphi}|=$ $|\langle\vec{k}, v\rangle|$ we see that equality holds if $\langle\vec{k}, v\rangle$ has a sign. 
We pass now to prove the second part of the statement. Using Cauchy-Schwarz inequality we get

$$
\begin{aligned}
\left(\sum_{i=1}^{N} \int_{\gamma^{i}}\left|\vec{k}^{i}\right| \mathrm{d} s\right)^{2} & =\left(\sum_{i=1}^{N} \frac{\int_{\gamma^{i}}\left|\vec{k}^{i}\right| \mathrm{d} s}{\sqrt{\ell\left(\gamma^{i}\right)}} \sqrt{\ell\left(\gamma^{i}\right)}\right)^{2} \\
& \leq\left(\sum_{i=1}^{N} \frac{\left(\int_{\gamma^{i}}\left|\vec{k}^{i}\right| \mathrm{d} s\right)^{2}}{\ell\left(\gamma^{i}\right)}\right)\left(\sum_{i=1}^{N} \ell\left(\gamma^{i}\right)\right) .
\end{aligned}
$$

Moreover by Hölder inequality

$$
\left(\int_{\gamma^{i}}\left|\vec{k}^{i}\right| \mathrm{d} s\right)^{2} \leq \ell\left(\gamma^{i}\right) \int_{\gamma^{i}}\left|\vec{k}^{i}\right|^{2} \mathrm{~d} s,
$$

that combined with (5.2) gives

$$
\begin{aligned}
\mathcal{E}\left(\cup_{i=1}^{N} \gamma^{i}\right) & =\sum_{i=1}^{N}\left(\int_{\gamma^{i}}\left|\vec{k}^{i}\right|^{2} \mathrm{~d} s+\ell\left(\gamma^{i}\right)\right) \geq \sum_{i=1}^{N}\left(\frac{\left(\int_{\gamma^{i}}\left|\vec{k}_{i}\right| \mathrm{d} s\right)^{2}}{\ell\left(\gamma^{i}\right)}+\ell\left(\gamma^{i}\right)\right) \\
& \geq \frac{\left(\sum_{i=1}^{N} \int_{\gamma^{i}}\left|\vec{k}_{i}\right| \mathrm{d} s\right)^{2}}{\sum_{i=1}^{N} \ell\left(\gamma^{i}\right)}+\sum_{i=1}^{N} \ell\left(\gamma^{i}\right) \geq \frac{\alpha^{2}}{\sum_{i=1}^{N} \ell\left(\gamma^{i}\right)}+\sum_{i=1}^{N} \ell\left(\gamma^{i}\right) \geq 2 \alpha .
\end{aligned}
$$

Lemma 5.4 We have that $I_{\mathcal{D}}$ defined in (5.1) equals zero if and only if the graph $G$ is stratified-straight.

Moreover, suppose that one of the following conditions holds:

(i) at every vertex $p$ the convex hull of the outer tangents contains the origin in its interior, that is $0 \in \operatorname{Int}\left(\operatorname{co}\left(\left\{d_{z, i}\right\}_{(z, i) \in \pi^{-1}(p)}\right)\right)$,

(ii) two edges $E_{i}$ and $E_{j}$ have both vertices in common and, if $\pi\left(z_{i}, i\right)=\pi\left(z_{j}, j\right)$ for some $z_{i}, z_{j}$, then $d^{z_{i}, i}$ and $d^{z_{j}, j}$ are linearly independent,

then $I_{\mathcal{D}}>0$.

Proof Let us prove the first claim. Suppose first that $I_{\mathcal{D}}=0$. Then there exists a sequence of networks $\left\{\mathcal{N}_{n}\right\}$ with $\widetilde{\mathcal{E}}\left(\mathcal{N}_{n}\right) \rightarrow 0$, and in particular $L\left(\mathcal{N}_{n}\right) \rightarrow 0$. This means that the networks $\mathcal{N}_{n}$ are converging strongly, up to translation, to a constant network $\mathcal{N}_{\infty}$, where every curve is collapsed to a point. In particular, recalling Definition 2.4, $\operatorname{Sing}\left(\mathcal{N}_{\infty}\right)=G$. By Proposition 3.9 we obtain that $\mathcal{N}_{\infty}$ belongs to $\mathcal{C}_{\text {Deg }}$. Recalling Definition 3.8, this implies that $G=\operatorname{Sing}\left(\mathcal{N}_{\infty}\right)$ is stratified straight. This proves one implication. On the other hand if $G$ is stratified straight, there exists a degenerate immersion $\Sigma_{0}$ whose regular curves are straight segments. By Proposition 4.2 there exists a sequence $\left\{\mathcal{N}_{n}\right\}$ of regular networks such that $\Gamma_{n} \rightarrow \Sigma_{0}$ in $H^{2}$. Analogously there exists recovery sequences for every rescaling $\frac{1}{\lambda} \Sigma_{0}$ of $\Sigma_{0}$ with 
$\lambda>>1$. By a diagonal argument one can find a sequence of regular networks whose energy converges to zero. This proves the opposite implication.

Consider any regular network $\mathcal{N}=(G, \Gamma)$ with $G$ graph with assigned angles encoded by $\mathcal{D}$.

Suppose now that i) holds. Since the number of vertices is finite there exists at least one junction $p \in \Gamma\left(V_{G}\right)$ that lies on the boundary of the convex hull of $\Gamma\left(V_{G}\right)$, and thus that lies on $\partial H$ where $H$ is a closed half plane containing $\Gamma\left(V_{G}\right)$. Then at least one of the curves arriving at $p$, call it $\gamma$, must have an outer tangent that goes out of $H$ and whose smallest angle with $\partial H$ is at least $c(\mathcal{D})$ where $c(\mathcal{D})>0$ depends only on $\mathcal{D}$ and it is strictly positive by assumption. Then, by Lemma 5.3, we have $\int_{\gamma}|\vec{k}| \mathrm{d} s \geq c(\mathcal{D})$ and thus $\mathcal{E}(\gamma) \geq \frac{\left(\int_{\gamma}|\vec{k}| \mathrm{d} s\right)^{2}}{\ell\left(\gamma^{i}\right)}+\ell\left(\gamma^{i}\right) \geq \frac{c(\mathcal{D})^{2}}{\ell\left(\gamma^{i}\right)}+\ell\left(\gamma^{i}\right) \geq 2 c(\mathcal{D})$.

Suppose now that ii) holds. We then have that the least positive angle $\delta \in[0,2 \pi)$ between $d^{z_{i}, i}$ and $d^{z_{j}, j}$ is actually strictly positive, i.e., $\delta>0$. Consider the two maps $\gamma^{i}:=\Gamma_{\mid E_{i}}$ and $\gamma^{j}:=\Gamma_{\mid E_{j}}$, with $\Gamma$ a competitor for $I_{\mathcal{D}}$. Then by Lemma 5.3 we have that $\int_{\gamma^{i}}\left|\vec{k}^{i}\right| \mathrm{d} s+\int_{\gamma^{j}}\left|\vec{k}^{j}\right| \mathrm{d} s \geq \delta$, and thus $\mathcal{E}\left(\gamma^{i}\right)+\mathcal{E}\left(\gamma^{j}\right) \geq 2 \delta$.

As another application of Lemma 5.3 we improve the lower bound (cf [8, Lemma 2.5]) of the elastic energy of a Theta-network that we introduced above in Definition 8.

Lemma 5.5 Let $\Theta$ be a Theta-network. Then

$$
\mathcal{E}(\Theta) \geq \frac{16 \pi}{3}
$$

Proof In order to prove the required lower bound we can assume that the curves $\gamma^{1}, \gamma^{2}, \gamma^{3}$ realize a minimizing Theta-network, which exists by [8, Theorem 4.10]. Then the curves $\gamma^{i}$ are injective and real analytic by [8, Proposition 4.11] and by Proposition A.3. Without loss of generality we let

$$
\gamma^{1}(0)=\gamma^{2}(0)=\gamma^{3}(0)=(0,0) \quad \text { and } \quad \gamma^{1}(1)=\gamma^{2}(1)=\gamma^{3}(1)=(a, 0),
$$

for some $a>0$.

We denote by $\alpha^{i}$ the angles between $(1,0)$ and $\tau^{i}(0)$. Up to relabeling the curves and up to a symmetry with respect to the horizontal axis we can assume that $\alpha^{1} \in\left[0, \frac{\pi}{3}\right]$ and that

$$
\alpha^{2}=\alpha^{1}+\frac{2}{3} \pi, \quad \alpha^{3}=\alpha^{1}-\frac{2}{3} \pi
$$

We claim that for any $i$ there exists $t^{i} \in[0,1]$ such that $\frac{\dot{\gamma}^{i}\left(t^{i}\right)}{\left|\dot{\gamma}^{i}\left(t^{i}\right)\right|}=(1,0)$. We suppose for now that the claim is proven, and proceed to show how to conclude. We apply Lemma 5.3 separately to $\left(\left.\gamma^{i}\right|_{\left[0, t^{i}\right]}\right)_{i=1}^{3}$ and to $\left(\left.\gamma^{i}\right|_{\left[t^{i}, 1\right]}\right)_{i=1}^{3}$. The total angle spanned by the three curves in the first case is 


$$
\begin{aligned}
\left|\alpha^{1}\right|+\left|\alpha^{2}\right|+\left|\alpha^{3}\right| & =\left|\alpha^{1}\right|+\left|\alpha^{1}+\frac{2}{3} \pi\right|+\left|\alpha^{1}-\frac{2}{3} \pi\right| \\
& =\alpha^{1}+\left(\alpha^{1}+\frac{2}{3} \pi\right)+\left(\frac{2}{3} \pi-\alpha^{1}\right) \\
& =\alpha^{1}+\frac{4}{3} \pi \geq \frac{4}{3} \pi
\end{aligned}
$$

and therefore by Lemma 5.3 the total energy of the first three pieces of curves $\left(\left.\gamma^{i}\right|_{\left[0, t^{i}\right]}\right)_{i=1}^{3}$ is at least $\frac{8}{3} \pi$. In a totally analogous way we conclude the same for the final pieces of curves $\left(\left.\gamma^{i}\right|_{\left[t^{i}, 1\right]}\right)_{i=1}^{3}$ and thus we conclude that $\mathcal{E}(\Theta) \geq \frac{16}{3} \pi$.

We are left to show how to prove the claim, namely that for any $i$ there exists $t^{i} \in[0,1]$ such that $\frac{\dot{\gamma}^{i}\left(t^{i}\right)}{\left|\dot{\gamma}^{i}\left(t^{i}\right)\right|}=(1,0)$. Unless the curve $\gamma^{i}$ is a horizontal straight segment (and in this case the result trivially follows) by analiticity there are finitely many intersections with the horizontal axis $\left(a_{j}, 0\right)=\gamma^{i}\left(t_{j}\right)$ with $t_{j}<t_{j+1}$. Moreover, since $\gamma^{i}(1)=(a, 0)$ with $a>0$, there exists an index $j$ such that $a_{j}<a_{j+1}$. We consider the arc $\sigma(t):=\gamma^{i}(t)$ with $t \in\left(t_{j}, t_{j+1}\right)$ that, without loss of generality, lies in the half plane $\{y>0\}$. It is possible to complete $\sigma(t)$ to a smooth simple closed curve with $t \in\left[t_{j}, s\right]$ for some $s>t_{j+1}$ such that for every $t \in\left(t_{j+1}, s\right)$ it holds $\frac{\dot{\sigma}(t)}{|\dot{\sigma}(t)|} \neq(1,0)$. Then by Hopf's Umlaufsatz [1, Theorem 2.4.7] the degree of the tangent map of $\sigma(t)$ is different from zero, and thus the tangent map is surjective. Therefore the value $(1,0)$ must be attained in the interval $\left[t_{j}, t_{j+1}\right]$ and the claim is proved.

\section{A Characterization of Degenerate Networks in $\mathbb{R}^{2}$}

Although the definition of the class of degenerate networks given in Definition 3.8 is very neat and convenient, it has a disadvantage: it is based on the validation of the angle condition given by Definition 3.2, and thus on the assumption that there exists a family $\mathcal{T}$ of real or virtual tangents. In general one cannot easily verify this condition. At least when the ambient space is $\mathbb{R}^{2}$ we are able to give an equivalent characterization based on conditions that can be validated by a procedure consisting in a finite number of steps. The procedure can be roughly summarized as follows: given an $N$-graph $G$ with assigned angles $\mathcal{D}$, to every path $\mathcal{P}$ made of consecutive edges in $G$ we assign an abstract "total turning angle" $\Theta(\mathcal{P})$ that is obtained by summing the turning angles (forced by the angle condition $\mathcal{D}$ ) at every junction of the path. This angle only depends on the graph structure and on $\mathcal{D}$, but not on a realization of the graph as a network. Then a network $\mathcal{N}=(G, \Sigma)$ satisfies the angle condition given by $\mathcal{D}$ if and only if: for every cycle $C$ (i.e., closed path) entirely contained in $\operatorname{Sing}(\mathcal{N})$ we have that $\Theta(C)$ is a multiple of $2 \pi$; for every (open) path $\mathcal{P}$ whose first and last edges are in $\operatorname{Reg}(\mathcal{N})$ but whose other edges are all in $\operatorname{Sing}(\mathcal{N})$ the total angle $\Theta(\mathcal{P})$ coincides with the angle between the real tangents (given by $\Sigma$ ) at the the second and penultimate vertices of the path.

From now on we fix the dimension of the ambient space to $d=2$. 
Definition 6.1 (Path and cycle) Let $G$ be an $N$-graph with assigned angles. A path composed of $\mathcal{J}$ edges is a sequence

$$
\begin{aligned}
\mathcal{P}:\{1, \ldots, \mathcal{J}\} & \rightarrow\{0,1\} \times\{1, \ldots, N\} \\
j & \rightarrow\left(z_{j}, i_{j}\right)
\end{aligned}
$$

such that for every $j \in\{1, \ldots, \mathcal{J}-1\}$ it holds $\pi\left(1-z_{j}, i_{j}\right)=\pi\left(z_{j+1}, i_{j+1}\right)$. A path is a cycle if $\pi\left(1-z_{\mathcal{J}}, i_{\mathcal{J}}\right)=\pi\left(z_{1}, i_{1}\right)$. If a path is not a cycle we call it open path.

We should think of $\left(z_{j}, i_{j}\right)$ as encoding the following fact: when we travel through the path, the $j$ th edge is the one with index $i_{j}$ and its first endpoint that we meet is $z_{j}$.

When the first element of a path $\mathcal{P}$ is $(z, i)$ and the last is $(w, j)$ sometimes we will simply say that $\mathcal{P}$ is a path from the edge $E_{i}$ to the edge $E_{j}$.

Let $\vec{a}$ and $\vec{b}$ two vectors in $\mathbb{R}^{2}$. Then the angle between $\vec{a}$ and $\vec{b}$, denoted by $\angle \vec{a}, \vec{b}$, is an element of $\mathbb{R} \bmod 2 \pi$. From now on and for the rest of the section, an angle $\angle \vec{a}, \vec{b}$ will always be understood as an element of $\mathbb{R} \bmod 2 \pi$, and a representative for $\angle \vec{a}, \vec{b}$ will be always assumed to be the element of $[0,2 \pi)$ such that the counterclockwise rotation of $\vec{a}$ of such an angle yields $\vec{b}$.

Definition 6.2 (Angle of a path) Let $G$ be an $N$-graph with assigned angles $\mathcal{D}$ in $\mathbb{R}^{2}$. We define the angle of a path $\mathcal{P}$ composed of $\mathcal{J}$ edges as

$$
\Theta(\mathcal{P}):= \begin{cases}\sum_{j=1}^{\mathcal{J}-1}\left(\angle-d^{1-z_{j}, i_{j}}, d^{z_{j+1}, i_{j+1}}\right) \bmod 2 \pi & \text { if } \mathcal{P} \text { is an open path } \\ \sum_{j=1}^{\mathcal{J}}\left(\angle-d^{1-z_{j}, i_{j}}, d^{z_{j+1}, i_{j+1}}\right) \bmod 2 \pi & \text { if } \mathcal{P} \text { is a cycle }\end{cases}
$$

with the understanding that the indices are modulo $\mathcal{J}$, so that $\mathcal{J}+1=1$.

For the sake of readability when we consider a path composed of $\mathcal{J}$ edges we often suppose that, up to relabeling and reparametrizing the edges, we have

$$
\begin{aligned}
\mathcal{P}:\{1, \ldots, \mathcal{J}\} & \rightarrow\{0,1\} \times\{1, \ldots, \mathcal{J}\} \\
j & \rightarrow(0, j)
\end{aligned}
$$

and that $\pi(1, j)=\pi(0, j+1)$. Moreover if $\mathcal{P}$ is a cycle we require that $\pi(1, \mathcal{J})=$ $\pi(0,1)$. In this setting

$$
\Theta(\mathcal{P}):= \begin{cases}\sum_{j=1}^{\mathcal{J}-1}\left(\angle-d^{1, j}, d^{0, j+1}\right) \bmod 2 \pi & \text { if } \mathcal{P} \text { is an open path } \\ \sum_{j=1}^{\mathcal{J}}\left(\angle-d^{1, j}, d^{0, j+1}\right) \bmod 2 \pi & \text { if } \mathcal{P} \text { is a cycle }\end{cases}
$$

with the understanding that the indices are modulo $\mathcal{J}$, so that $\mathcal{J}+1=1$. When $\mathcal{N}=(G, \Gamma)$ is a network of class $\mathcal{C}_{\text {Reg }}$ and we denote by $\gamma^{j}=\left.\Gamma\right|_{E_{j}}$ we have that $\gamma^{j}(1)=\gamma^{j+1}(0)$ for $j \in\{1 \ldots, \mathcal{J}-1\}$ and, if $\mathcal{P}$ is a cycle, $\gamma^{\mathcal{J}}(1)=\gamma^{1}(0)$.

Lemma 6.3 Let $\left\{\mathcal{N}_{n}\right\}_{n \in \mathbb{N}}$ be a sequence of networks in $\mathcal{C}_{\mathrm{Reg}}$ such that

$$
\limsup _{n} \mathcal{E}\left(\mathcal{N}_{n}\right) \leq C<+\infty
$$


- Suppose that $\mathcal{P}$ is an open path composed of $\mathcal{J}$ edges and that $\lim _{n \rightarrow \infty} \ell\left(\gamma_{n}^{j}\right)=0$ for $j \in\{2, \ldots, \mathcal{J}-1\}$. Then $\Theta(\mathcal{P})=\lim _{n \rightarrow \infty}\left(\angle-\tau_{n}^{1,1}, \tau_{n}^{0, \mathcal{J}}\right)$.

- Suppose that $\mathcal{P}$ is a cycle composed of $\mathcal{J}$ edges and that $\lim _{n \rightarrow \infty} \ell\left(\gamma_{n}^{j}\right)=0$ for $j \in\{1, \ldots, \mathcal{J}\}$. Then $\Theta(\mathcal{P})=0(\bmod 2 \pi)$.

Proof Suppose that $\mathcal{P}$ is an open path. For every $n \in \mathbb{N}$ we have that

$$
\begin{aligned}
\angle-\tau_{n}^{1,1}, \tau_{n}^{0, \mathcal{J}} & =\sum_{j=1}^{\mathcal{J}-1} \angle-\tau_{n}^{1, j}, \tau_{n}^{0, j+1}+\sum_{j=2}^{\mathcal{J}-1} \angle \tau_{n}^{0, j},-\tau_{n}^{1, j} \\
& =\sum_{j=1}^{\mathcal{J}-1}\left(\angle-d^{1, j}, d^{0, j+1}\right)+\sum_{j=2}^{\mathcal{J}-1} \angle \frac{\dot{\gamma}_{n}^{j}(0)}{\left|\dot{\gamma}_{n}^{j}(0)\right|}, \frac{\dot{\gamma}_{n}^{j}(1)}{\left|\dot{\gamma}_{n}^{j}(1)\right|} .
\end{aligned}
$$

Then thanks to Lemma 3.1 one gets the desired result passing to the limit $n \rightarrow \infty$.

If instead $\mathcal{P}$ is a cycle, then $\tau_{n}^{0, \mathcal{J}+1}=\tau_{n}^{0,1}$ and so using again by Lemma 3.1 we obtain

$$
\Theta(\mathcal{P})=\lim _{n \rightarrow \infty} \angle-\tau_{n}^{1,1}, \tau_{n}^{0,1}=0
$$

as desired.

It is possible to give an alternative definition of the angle condition for a singular network with respect to Definition 3.2 in term of paths, justified by Lemma 6.3.

Definition 6.4 (Angle condition for a singular network in term of paths) Let $G$ be an $N$-graph with assigned angles. We say that a network $\mathcal{N}$ satisfies the angle condition if

(i) its regular curves satisfy the angle condition in the sense of Definition 2.11;

(ii) if $\mathcal{P}$ is a cycle composed of $\mathcal{J}$ edges such that for every $j \in\{1, \ldots, \mathcal{J}\}$ the edges $E_{j}$ are in $\operatorname{Sing}(\mathcal{N})$ then $\Theta(\mathcal{P})=0(\bmod 2 \pi)$;

(iii) if $\mathcal{P}$ is an open path composed of $\mathcal{J}$ edges such that for every $j \in\{2, \ldots, \mathcal{J}-1\}$ the edges $E_{j}$ are in $\operatorname{Sing}(\mathcal{N})$ and $E_{1} \cup E_{\mathcal{J}} \subset \operatorname{Reg}(\mathcal{N})$ then $\Theta(\mathcal{P})=\angle-\tau^{1,1}, \tau^{0, \mathcal{J}}$.

Remark 6.5 Condition (i) can be seen as a particular case of (iii) by taking $\mathcal{J}=2$.

Clearly one has to modify also Definitions 3.6 and 3.8 accordingly to the above new definition of angle condition.

Definition 6.6 Fix an $N$-graph with assigned angles $G$. A subgraph $H \subseteq G$ is stratified-straight if there exists a finite sequence of subgraphs (called strata)

$$
\emptyset=H_{q} \subset H_{q-1} \subset \ldots \subset H_{1} \subset H_{0}=H
$$

and maps $\Sigma_{i}: H_{i} \rightarrow \mathbb{R}^{2}$ such that for $i=0, \ldots, q-1$ 
- $\left(H_{i}, \Sigma_{i}\right)$ is a (possibly singular) network that satisfies the angle condition in the sense of Definition 6.4 and whose curves are (possibly degenerate) straight segments;

- $H_{i+1}=\operatorname{Sing}\left(\left(H_{i}, \Sigma_{i}\right)\right)$.

We call step of $G$ the minimal $q$ for which the above holds.

Definition 6.7 Let $G$ be an $N$-graph with assigned angles. A network $\mathcal{N}=(G, \Gamma)$ is degenerate if

- it satisfies the angle condition in the sense of Definition 6.4;

- the singular part $\operatorname{Sing}(\mathcal{N})$ is a stratified-straight subgraph in the sense of Definition 6.6.

Proposition 6.8 Let $G$ be a graph with assigned angles. Suppose that the ambient space is $\mathbb{R}^{2}$. Then a network $\mathcal{N}=(G, \Gamma)$ satisfies Definition 6.4 if and only if it satisfies Definition 3.2 for some choice of virtual tangent vectors.

Proof Suppose that $\mathcal{N}$ satisfies the angle condition as in Definition 6.4. The outward tangents of the regular curves of $\mathcal{N}$ satisfy the requests of Definition 3.2 because of condition (i) in Definition 6.4. We have to construct the set of virtual tangents. We assume for the moment that at least one curve of $\mathcal{N}$ is regular, for instance $\Gamma_{\mid E_{1}}=$ : $\gamma^{1}$. Let $H \subset G$ be a closed connected component of $\operatorname{Sing} \mathcal{N}$ and suppose that an endpoint $p_{0}=\pi(0,1)$ of $E_{1}$ lies in $H$. We define $\tau^{w, j}$ for any $\pi(w, j)=p_{0}$ with $\gamma^{j}$ singular by setting $\tau^{w, j}$ equal to the counterclockwise rotation of $\tau^{0,1}$ of the angle $\angle d^{0,1}, d^{w, j}$; whenever such $\tau^{w, j}$ has been defined, we also set $\tau^{1-w, j}=-\tau^{w, j}$. It follows that (3.1) is satisfied at $p_{0}$. Observe that if $\tau^{z, i}, \tau^{y, l}$ are defined by this last step and $\pi(z, i)=\pi(y, l) \neq p_{0}$, then

$$
\angle \tau^{z, i}, \tau^{y, l}=\angle d^{z, i}, d^{y, l} .
$$

In fact the path $\mathcal{P}$ given by

$$
\mathcal{P}(1)=(1-z, i), \quad \mathcal{P}(2)=(y, l),
$$

is a cycle contained in $\operatorname{Sing} \mathcal{N}$, and thus by assumption we get that

$$
\begin{aligned}
\Theta(\mathcal{P}) & =\angle-d^{z, i}, d^{y, l}+\angle-d^{1-y, l}, d^{1-z, i}=\angle-d^{z, i}, d^{y, l}+\angle-\tau^{1-y, l}, \tau^{1-z, i}= \\
& =\angle-d^{z, i}, d^{y, l}+\angle \tau^{y, l},-\tau^{z, i}=\pi+\angle d^{z, i}, d^{y, l}+\pi+\angle \tau^{y, l}, \tau^{z, i}= \\
& =2 \pi+\angle d^{z, i}, d^{y, l}+2 \pi-\angle \tau^{z, i}, \tau^{y, l}=0 \bmod 2 \pi .
\end{aligned}
$$

With this procedure we have defined every virtual tangent at $p_{0}$ of singular edges with an endpoint at $p_{0}$, and some virtual tangents at $p \neq p_{0}$ of singular edges having endpoints at $p$ and $p_{0}$.

Let $p \neq p_{0}$ be now any vertex at which at least one virtual or real tangent $\tau^{z, i}$ is defined. We can perform the very same construction for the still undefined virtual tangents at $p$ using rotations of $\tau^{z, i}$ in place of the original $\tau^{0,1}$. By (6.1) it follows that (3.1) is satisfied at $p$. 
Notice that if $\pi(x, a)=\pi(z, i) \neq p_{0}, \pi(y, b)=\pi(1-z, i) \neq p_{0}$, and $\pi(1-$ $x, a)=\pi(1-y, b)=p_{0}$ (and thus we have just constructed $\tau^{z, i}$ as a rotation of $\tau^{x, a}$ and $\tau^{1-z, i}$ as a rotation of $\left.\tau^{y, b}\right)$, then $\tau^{z, i}=-\tau^{1-z, i}$ as desired. In fact considering the cycle

$$
\mathcal{P}(1)=(1-y, b), \quad \mathcal{P}(2)=(1-z, i), \quad \mathcal{P}(3)=(x, a),
$$

we get that

$$
\Theta(\mathcal{P})=\angle-\tau^{y, b}, \tau^{1-z, i}+\angle-\tau^{z, i}, \tau^{x, a}+\angle-\tau^{1-x, a}, \tau^{1-y, b}=0 \bmod 2 \pi .
$$

On the other hand, since the sum of the exterior angles of a triangle equals $2 \pi$, we have that

$$
\angle-\tau^{y, b},-\tau^{z, i}+\angle-\tau^{z, i}, \tau^{x, a}+\angle-\tau^{1-x, a}, \tau^{1-y, b}=2 \pi=0 \bmod 2 \pi,
$$

and subtracting (6.3) to (6.2) we get that $\angle-\tau^{y, b}, \tau^{1-z, i}=\angle-\tau^{y, b},-\tau^{z, i} \bmod 2 \pi$. Now if $p_{1} \in H$ is a vertex of an edge having the other endpoint at $p_{0}$, all the (virtual and real) tangents at $p_{1}$ are defined. From the above argument, it follows that we can define $\tau^{1-w, j}=-\tau^{w, j}$ for any virtual tangent $\tau^{w, j}$ of a singular edge $E_{j}$ having an endpoint at $p_{1}$ without getting contradictions with the other already defined virtual tangents.

Therefore it follows that we can then iterate the above arguments, possibly considering cycles passing through $p_{0}$ that are not triangles, and use the assumption on $\Theta(\mathcal{P})$, in order to check that the iterated construction of virtual tangents does not lead to contradictions. Eventually, we are able to define the virtual tangents at any vertex of the connected component $H$, getting that Definition 3.2 is satisfied at such vertices. We can apply the same argument to any connected component $H$ of $\operatorname{Sing} \mathcal{N}$, completing the implication.

If instead it occurs that $\operatorname{Sing} \mathcal{N}=G$, we can just choose $\tau^{0,1}=(1,0)=-\tau^{1,1}$ arbitrarily. Then we can perform the very same construction described above.

Conversely, suppose now that Definition 3.2 is satisfied. Then obviously the regular curves satisfy the angle condition (i) of Definition 6.4. Moreover, if $\mathcal{P}$ is an open path, then the condition $\tau^{z, i}=-\tau^{1-z, i}$ on the virtual tangents implies point iii) of Definition 6.4. More generally observe that if the edges seen by a path $\mathcal{P}$ of step $\mathcal{J}$ are contained in $\operatorname{Sing} \mathcal{N}$, and for simplicity we write $\mathcal{P}(i)=(0, i)$, then

$$
\sum_{l=l_{0}}^{L-1} \angle-\tau^{1, l}, \tau^{0, l+1}=\angle-\tau^{1, l_{0}}, \tau^{0, L} \bmod 2 \pi .
$$


So, finally suppose that $\mathcal{P}$ is a cycle in $\operatorname{Sing} \mathcal{N}$ and we write $\mathcal{P}(i)=(0, i)$ for simplicity; by the fact that every involved tangent is virtual, using (6.4) we get that

$$
\begin{aligned}
\Theta(\mathcal{P}) & =\left(\sum_{i=1}^{\mathcal{J}-1} \angle-\tau^{1, i}, \tau^{0, i+1}\right)+\angle-\tau^{1, \mathcal{J}}, \tau^{0,1}=\angle-\tau^{1,1}, \tau^{0, \mathcal{J}}+\angle-\tau^{1, \mathcal{J}}, \tau^{0,1}= \\
& =\angle \tau^{0,1}, \tau^{0, \mathcal{J}}+\angle \tau^{0, \mathcal{J}}, \tau^{0,1}=0 \bmod 2 \pi
\end{aligned}
$$

thus completing the equivalence of the definitions.

We underline the fact that in Definition 6.6 we simply replace the angle condition of Definition 3.2 by the one of Definition 6.4, and this replacement directly affects the new Definition 6.7 of degenerate networks. Then it is clear that taking advantage of the above proposition we are also able to prove the following:

Corollary 6.9 Let $G$ be a graph with assigned angles. Suppose that the ambient space is $\mathbb{R}^{2}$. Then a network $\mathcal{N}=(G, \Gamma)$ is degenerate in the sense of Definition 3.8 if and only if it is degenerate in the sense of Definition 6.7.

Remark 6.10 As we already mentioned, we remark again that it is somehow easier to use Definition 3.2 in the technical arguments. However, in the very remarkable case of dimension $d=2$, Definition 6.4 has the great advantage of being verifiable by an algorithm with finitely many steps. This is clearly not true for the general Definition 3.2.

\section{On the Relation Between Straight and Stratified Straight Subgraphs}

In this section we study a simple but remarkable case in which we completely characterize stratified straight or straight subgraphs. This helps us to better understand the algebraic and combinatorial relation between these two concepts, together with providing a non-trivial case in which the two definitions are not equivalent.

In the whole section we study networks in $\mathbb{R}^{2}$ and we take advantage of the equivalent characterization of the class of degenerate networks we presented in Sect. 6: we use Definitions 6.4, 6.6 and 6.7.

Throughout the section we will consider an $N$-graph $G$ with junctions of order at most four and suppose that for every junction $p=\pi\left(z_{1}, i_{1}\right)=\ldots=\pi\left(z_{k}, i_{k}\right)$ with $k \leq 4$ the vectors $d^{z_{1}, i_{1}}, \ldots, d^{z_{k}, i_{k}}$ are distinct and they form angles that are multiples of $\frac{\pi}{2}$. In this section, if $\vec{a}, \vec{b}$ are two planar vectors, we denote by $\angle \vec{a}, \vec{b} \in[0,2 \pi)$ the angle described by the counterclockwise rotation of $\vec{a}$ that yields $\vec{b}$.

Remark 7.1 Let $\mathcal{N}=(G, \Gamma)$ be a degenerate network of the type considered above, $H$ a stratified straight subgraph of $G$ composed of edges $E_{1}, \ldots, E_{k}$ and $\Sigma: H \rightarrow \mathbb{R}^{2}$ such that $(H, \Sigma)$ is a (possibly singular) network that satisfies the angle condition in the sense of Definition 6.4 and whose curves are (possibly degenerate) straight segments. We observe that there exist only two possible orthogonal directions, identified by two orthogonal unit vectors $a, b$, such that, if $\sigma^{i}:=\left.\Sigma\right|_{E_{i}}$ is a regular straight segment, then $\dot{\sigma}^{i}$ is parallel to $a$ or $b$. In particular, up to rotation, we can assume that $a=(1,0)$ and $b=(0,1)$. 
Remark 7.2 (Canonical assignment of the vectors $d^{z, i}$ ) Let $\mathcal{N}=(G, \Gamma)$ be a degenerate network and $H$ a stratified straight subgraph of $G$ composed of the edges $E_{1}, \ldots, E_{k}$. For every $(z, i) \in\{0,1\} \times\{1, \ldots, k\}$ we can give an explicit choice of the vector $d^{z, i}$, once a first edge is chosen. Fix first $d^{0,1}=(1,0)$ for example. For every $(z, i) \in\{0,1\} \times\{1, \ldots, k\}$ we want that $d^{z, i} \in\{( \pm 1,0),(0, \pm 1)\}$. We require then that for any $i$ it holds

$$
d^{0, i}=-d^{1, i}
$$

Then this choice is well defined for any $i$ and uniquely defines the $d^{z, i}$ s. In fact, by considering a path from $\pi(0,1)$ to $\pi(z, i)$ and using the rule (7.1) one can determine uniquely $d^{z, i}$. Suppose by contradiction that following two different paths $\mathcal{P}$ and $\mathcal{P}^{\prime}$ from $(0,1)$ to $(z, i)$ and using the rule (7.1) we get different results $d^{(z, i)}=a_{\mathcal{P}}$ and $d^{z, i}=b_{\mathcal{P}^{\prime}}$. Then $\angle a_{\mathcal{P}}, b_{\mathcal{P}^{\prime}} \in\left\{\frac{\pi}{2}, \pi, \frac{3 \pi}{2}\right\}$. Call $\mathcal{P}^{\prime \prime}$ the inverted path of $\mathcal{P}^{\prime}$ from $\pi(z, i)$ to $\pi(0,1)$. We can join the two paths $\mathcal{P}$ and $\mathcal{P}^{\prime \prime}$ and the resulting path is a cycle $\mathcal{Q}$ starting from $(0,1)$. Then it follows that $\Theta(\mathcal{Q}) \in\left\{\frac{\pi}{2}, \pi, \frac{3 \pi}{2}\right\}$, a contradiction to the fact that $H$ is stratified straight.

This canonical choice of the $d^{z, i}$ clearly depends on the choice of a starting vertex $\pi(0,1)$ together with the assignment $d^{0,1}=(0,1)$. We can use such canonical choice in order to introduce an order relation $\preceq$ on the set of vertices.

Definition 7.3 Let $H$ be a connected stratified straight subgraph of $G$, and suppose that $E_{i_{0}} \subset H$. Starting from $\pi\left(0, i_{0}\right)$ define $d^{0, i_{0}}=(1,0)$ and canonically assign the vectors $d^{z, i}$ as specified in Remark 7.2. Let $v$ and $w$ be two vertices of $H$. We say that $v \preceq w$ if and only if there exists a path $\mathcal{P}:\{1, \ldots, \mathcal{J}\} \rightarrow\{0,1\} \times\{1, \ldots, N\}$ such that

$$
\begin{aligned}
& \mathcal{P}(1)=\left(z_{1}, i_{1}\right), \mathcal{P}(\mathcal{J})=\left(z_{\mathcal{J}}, i_{\mathcal{J}}\right), \quad \text { with } v=\pi\left(z_{1}, i_{1}\right) \text { and } w=\pi\left(z_{\mathcal{J}}, i_{\mathcal{J}}\right), \\
& d^{\mathcal{P}(i)} \neq(-1,0) \quad \forall i=1, \ldots, \mathcal{J}-1,
\end{aligned}
$$

Also, we say that $v \prec w$ if and only if $v \preceq w$ and $w \npreceq v$.

Roughly speaking $v \preceq w$ if and only if we can reach $w$ starting from $v$ with a path that "never goes left". Also observe that the order relation depends on the choice of a certain edge $E_{i_{0}}$ in the considered subgraph.

The order relation defines two subsets of the vertices as defined below.

Definition 7.4 Let $H$ be a connected stratified straight subgraph of $G$, and suppose that $E_{i_{0}} \subset H$. Starting from $\pi\left(0, i_{0}\right)$ define $d^{0, i_{0}}=(1,0)$ and canonically assign the vectors $d^{z, i}$ as specified in Remark 7.2. We define

$$
\begin{aligned}
& X\left(i_{0}\right):=\left\{w \in V_{H} \mid \pi\left(0, i_{0}\right) \prec w\right\} \\
& Y\left(i_{0}\right):=V_{H} \backslash X\left(i_{0}\right) .
\end{aligned}
$$

Proposition 7.5 Let $G$ be an $N$-graph with assigned angles. Suppose that every junction of $G$ has order at most 4 and that, if $p$ is a junction with $\pi^{-1}(p)=$ 
$\left\{\left(z_{1}, i_{1}\right), \ldots,\left(z_{k}, i_{k}\right)\right\}$, then the vectors $d^{z_{1}, i_{1}}, \ldots, d^{z_{k}, i_{k}}$ form angles equal to $\frac{n \pi}{2}$ for $n \in\{1,2,3\}$. Let $H \subset G$ be a connected stratified straight subgraph and denote by $\left(H, \Sigma_{0}\right)$ the first stratum of $H$.

Suppose that for every $E_{i_{0}} \subset H \cap \operatorname{Sing}\left(H, \Sigma_{0}\right)$ there do not exist cycles $\mathcal{P}=$ $(\mathcal{P}(1), \ldots, \mathcal{P}(\mathcal{J}))$ such that

$$
\begin{aligned}
& \mathcal{P}(1)=\left(z_{0}, i_{0}\right) \\
& d^{\left(z_{0}, i_{0}\right)}=(1,0) \\
& d^{\mathcal{P}(j)} \neq(-1,0) \quad \forall j=2, \ldots, \mathcal{J}-1 .
\end{aligned}
$$

Then $H$ is straight.

Proof Denote by $\left(H, \Sigma_{0}\right)$ the first stratum of $H$, i.e. $\Sigma_{0}: H \rightarrow \mathbb{R}^{2}$ defines a degenerate network, the regular curves of $\Sigma_{0}$ are straight segments and at least one curve, say $\sigma_{0}^{i_{0}}:=\left.\Sigma_{0}\right|_{E_{i_{0}}}$, is degenerate. We denote by $\sigma_{0}^{i}$ the curve $\left.\Sigma_{0}\right|_{E_{i}}$.

We want to prove that we can modify $\left(H, \Sigma_{0}\right)$ into a new degenerate network $\left(H, \tilde{\Sigma}_{0}\right)$ such that $\tilde{\sigma}_{0}^{i_{0}}$ is a regular straight segment and if $\sigma_{0}^{i}$ is a regular straight segment then so is $\tilde{\sigma}_{0}^{i}$. In such a way, since the edges of $H$ are finitely many, iterating the argument we conclude that $H$ is straight.

In order to simplify the notation, let us write that $i_{0}=1$.

Fix $d^{0,1}=(1,0)$ and consider the order relation $\preceq$ induced by this choice as given by Definition 7.3. Clearly $Y(1) \neq \varnothing$, in fact $\pi(0,1) \nprec \pi(0,1)$ by definition. Also we have that $\pi(1,1) \in X(1) \neq \emptyset$; indeed $\pi(0,1) \preceq \pi(1,1), \sigma_{0}^{1}$ is degenerate, and if by contradiction $\pi(1,1) \preceq \pi(0,1)$ then there exists a path $\mathcal{P}$ of step $\mathcal{J}$ that for simplicity we denote by

$$
\begin{aligned}
& \mathcal{P}(1)=\left(0, k_{0}\right), \\
& \mathcal{P}(j)=(0, j) \quad \forall j=2, \ldots, \mathcal{J}-1, \\
& \mathcal{P}(\mathcal{J})=(0,1),
\end{aligned}
$$

such that $\pi\left(0, k_{0}\right)=\pi(1,1)$ and $d^{0, j} \neq(-1,0)$ for any $j=k_{0}, 2, \ldots, \mathcal{J}-1$. But since $d^{0,1}=(1,0)$, the cycle given by

$$
\begin{aligned}
& \mathcal{R}(1)=(0,1), \\
& \mathcal{R}(j)=\mathcal{P}(j-1) \quad \forall j=2, \ldots, \mathcal{J},
\end{aligned}
$$

contradicts the hypothesis.

Now we construct $\tilde{\Sigma}_{0}$. Let us first define such map on the set of vertices $V_{H}$ by setting

$$
\tilde{\Sigma}_{0}(v)= \begin{cases}\Sigma_{0}(v) & \text { if } v \in Y(1), \\ \Sigma_{0}(v)+(\varepsilon, 0) & \text { if } v \in X(1),\end{cases}
$$


for some $\varepsilon>0$. We need to check that we can extend $\tilde{\Sigma}_{0}$ to the edges in a consistent way. We claim that for any edge $E_{j}$, if $\tilde{\Sigma}_{0}^{j}(0) \neq \tilde{\Sigma}_{0}^{j}(1)$, the images of its boundary points $\tilde{\Sigma}_{0}^{j}(0), \tilde{\Sigma}_{0}^{j}(1)$ can be connected by a regular straight segment $\tilde{\sigma}_{0}^{j}(t)$ such that $d^{0, j}=\alpha\left(\tilde{\sigma}_{0}^{j}(1)-\tilde{\sigma}_{0}^{j}(0)\right)$ with $\alpha>0$. Assuming that the claim is true, then the map $\tilde{\Sigma}_{0}$ is defined on every edge in the natural way by connecting with a straight segment the image through $\tilde{\Sigma}_{0}$ of its endpoints. For $\varepsilon$ small enough, all the regular straight segments of $\Sigma_{0}$ remain regular. Moreover, since $\pi(1,1) \in X(1)$ and $\pi(0,1) \in Y(1)$ then $\tilde{\sigma}_{0}^{1}(0) \neq \tilde{\sigma}_{0}^{1}(1)$ and thus $\tilde{\sigma}_{0}^{1}$ is a regular straight segment, and the proof is completed.

In order to prove the claim we distinguish two cases, adopting the following notation:

$$
\begin{aligned}
& A(1)=\left\{E_{i} \text { edge of } H \mid \pi(0, i) \in X(1), \pi(1, i) \in X(1)\right\}, \\
& B(1)=\left\{E_{i} \text { edge of } H \mid \pi(0, i) \notin X(1), \pi(1, i) \notin X(1)\right\}, \\
& C(1)=\left\{E_{i} \text { edge of } H\right\} \backslash(A(1) \cup B(1)) .
\end{aligned}
$$

Case 1: Assume first that $E_{j} \in A(1) \cup B(1)$. Then both endpoints of $E_{j}$ have been moved or both remained unchanged, that is

$$
\tilde{\Sigma}_{0}(0)=\Sigma_{0}(0) \quad \text { and } \quad \tilde{\Sigma}_{0}(1)=\Sigma_{0}(1)
$$

or

$$
\tilde{\Sigma}_{0}(0)=\Sigma_{0}(0)+(\varepsilon, 0) \quad \text { and } \quad \tilde{\Sigma}_{0}(1)=\Sigma_{0}(1)+(\varepsilon, 0) .
$$

If $\Sigma_{0}(0)=\Sigma_{0}(1)$, that is $\sigma_{0}^{j}$ is degenerate, then $\tilde{\Sigma}_{0}(0)=\tilde{\Sigma}_{0}(1)$ as well and $\tilde{\sigma}_{0}^{j}$ will be degenerate. If otherwise $\Sigma_{0}(0) \neq \Sigma_{0}(1)$, that is $\sigma_{0}^{j}$ is a regular straight segment, then there is $\alpha>0$ such that $d^{0, j}=\alpha\left(\Sigma_{0}(1)-\Sigma_{0}(0)\right)$, and then $d^{0, j}=\alpha\left(\tilde{\Sigma}_{0}(1)-\tilde{\Sigma}_{0}(0)\right)$ as well and a straight segment $\tilde{\sigma}_{0}$ satisfies the claim.

Case 2: We are then left with the case of $E_{j} \in C(1)$, that is when one of the endpoints of $E_{j}$ has been moved and the other has not. In such a case, up to relabeling, we can assume $\pi(1, j) \in X(1)$ (i.e., $\pi(0,1) \prec \pi(1, j))$ and $\pi(0, j) \in Y(1)$. Thus

$$
\tilde{\Sigma}_{0}(\pi(0, j))=\Sigma_{0}(\pi(0, j)) \quad \text { and } \quad \tilde{\Sigma}_{0}(\pi(1, j))=\Sigma_{0}(\pi(1, j))+(\varepsilon, 0) .
$$

If $d^{0, j}=(1,0)$, the claim is proved. Let us show that this is the case. We have that $d^{0, j} \neq \pm(0,1)$, otherwise $E_{j}$ would belong to $A(1) \cup B(1)$, because $\pi(1, j) \preceq \pi(0, j) \preceq \pi(1, j)$. Suppose by contradiction that $d^{0, j}=-(1,0)$. In this case $d^{1, j}=-d^{0, j}=(1,0)$, and thus $\pi(1, j) \preceq \pi(0, j)$. By assumption there exists a path $\mathcal{P}$ with $\pi(\mathcal{P}(1))=\pi(0,1), \mathcal{P}(\mathcal{J})=(1, j)$ such that $d^{\mathcal{P}(i)} \neq(-1,0)$ for any $i=$ $1, \ldots, \mathcal{J}-1$. Extending $\mathcal{P}$ to a longer path by setting $\mathcal{P}(\mathcal{J}+1)=(0, j)$ it follows that $\pi(0,1) \preceq \pi(0, j)$. Actually we have that $\pi(0,1) \prec \pi(0, j)$, 
Fig. 10 In the picture we have the image in $\mathbb{R}^{2}$ of a regular network $(G, \Gamma)$

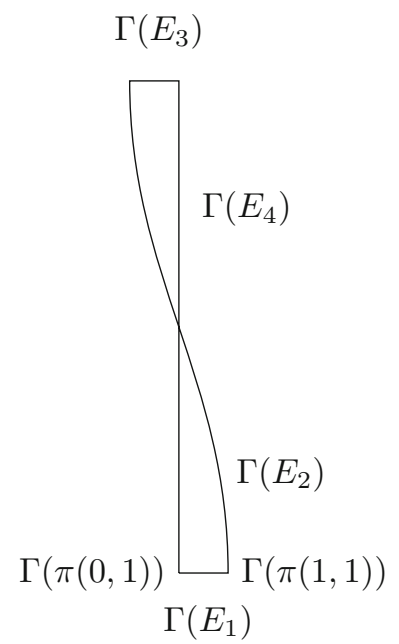

for otherwise, as shown before in the case of $\pi(1,1)$, we could construct a cycle $\mathcal{Q}$ of step $\mathcal{K}$ starting at $\mathcal{Q}(1)=(1, j)$ such that $d^{(1, j)}=(1,0)$ and $d^{\mathcal{Q}(k)} \neq(-1,0)$ for any $k=2, \ldots, \mathcal{K}-1$, contradicting the hypothesis. But then we have that $\pi(0, j) \in X(1) \cap Y(1)=\emptyset$, that is impossible. Therefore $d^{0, j} \neq(-1,0)$ and the proof of the claim, and then of the proposition, is completed.

The assumption of Proposition 7.5 has the advantage of being verifiable just by looking at all the possible cycles starting from the degenerate edges of a stratified straight graph. In the next example we show that the assumption of Proposition 7.5 is also necessary, and thus the statement of such proposition is sharp.

Example 7.6 Here we give a simple but remarkable example of a graph $G$ with assigned angles such that every junction of $G$ has order at most 4 and that, if $p$ is a junction with $\pi^{-1}(p)=\left\{\left(z_{1}, i_{1}\right), \ldots,\left(z_{k}, i_{k}\right)\right\}$, then the vectors $d^{z_{1}, i_{1}}, \ldots, d^{z_{k}, i_{k}}$ form angles equal to $\frac{n \pi}{2}$ with $n \in\{1,2,3\}$; moreover $G$ is stratified straight, but it is not straight, and in fact the assumption of Proposition 7.5 is violated.

Consider the image in $\mathbb{R}^{2}$ of the regular network drawn in Fig. 10.

Such graph $G$ is stratified straight, in fact one can easily construct a sequence of maps $\Gamma_{n}: G \rightarrow \mathbb{R}^{2}$ such that $\left(G, \Gamma_{n}\right)$ is regular and $\Gamma_{n}$ converges strongly in $H^{2}$ to a constant map, i.e., the image of the graph disappears with elastic energy going to zero. However $G$ is not straight and a possible stratification of $G$ is given by

$$
H_{0}=G, \quad H_{1}=E_{1} \cup E_{3} .
$$

A possible immersion $\Sigma_{1}$ of the stratum $H_{1}$ is given by two disjoint and horizontal straight segments. In the couple $\left(H_{0}, \Sigma_{0}\right)$, the immersion $\Sigma_{0}$ is given by four curves: $\gamma^{2}$ and $\gamma^{4}$ are two vertical and overlapping straight segments with the same endpoints, while $\gamma^{1}, \gamma^{3}$ are two constant maps, coinciding with the endpoints of $\gamma^{2}$ and $\gamma^{4}$. 
We remark that, in fact, the assumption of Proposition 7.5 is not satisfied. More generally we see that as long as a stratified straight subgraph $H$ contains a cycle like the one in Fig. 10, then $H$ is not straight, the assumption of Proposition 7.5 is not satisfied, and its proof does not work.

\section{Side Remarks}

\subsection{Curves on Surfaces/Manifolds}

We comment on the fact that the very same kind of definitions about degenerate networks characterize a suitably defined problem for networks of curves into a 2dimensional surface in $\mathbb{R}^{3}$.

Fix a 2-dimensional closed surface $S \subset \mathbb{R}^{3}$ and consider a network $\mathcal{N}=(G, \Gamma)$ with $\Gamma: G \rightarrow S$. Given a curve $\gamma: I \rightarrow S$, the geodesic curvature is given by

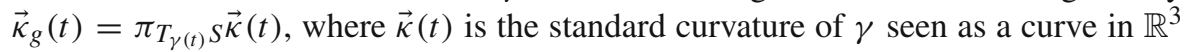
and $T_{x} S$ is the tangent plane to $S$ at the point $x \in S$. Therefore we define the general elastic energy of the curve $\gamma$ as

$$
\mathcal{E}_{\alpha, \beta}(\gamma):=\alpha \int_{\gamma}\left|\vec{\kappa}_{g}\right|^{2} \mathrm{~d} s+\beta L(\gamma) .
$$

Observe that, since the normal component of the curvature vector $\vec{\kappa}$ of $\gamma$ is bounded in terms of the second fundamental form of $S$, then a bound on $\mathcal{E}_{\alpha, \beta}$ actually is a bound on the $L^{2}$ norm of the whole vector $\vec{\kappa}$.

Analogous definitions of angle condition and degenerate network can be given for the class of networks having image in S. So, the compactness result of Proposition 3.9 can be still easily proved in this case. Then, also the proof of the recovery sequence presented in Proposition 4.2 can be adapted to the current situation. Maintaining the notation of the proof of Proposition 4.2, let us say that $H_{0} \subset \operatorname{Sing} \mathcal{N}$ is connected and $\Gamma\left(H_{0}\right)=p \in S$; we want to provide an immersion of $H_{0}$ in $S$. Since $S$ is a surface, there exists a local chart $\varphi: U \rightarrow \mathbb{R}^{2}$ at $p$ such that $\varphi$ is isothermal, i.e., the metric tensor $g$ of $S$ can be expressed as $g_{i j}=\lambda^{2} \delta_{i j}$ on $U$ in the chart $\varphi$. In particular we have that $\varphi$ is a conformal diffeomorphism with its image and its differential preserves angles between tangent vectors. Hence we can construct immersions of $H_{0}$ in $\varphi(U)$ exactly as in Proposition 4.2 and then we get the desired recovery sequence by applying $\varphi^{-1}$.

\subsection{Fixed Length}

We briefly discuss here an easier variant of Problem 2.13 of some interest in the applications.

Remark 8.1 (Fixed length) Putting $\alpha=1$ and $\beta=0$ in (2.3), then $\widetilde{\mathcal{E}}$ reduces to a functional that we denote by $\mathcal{W}$, by analogy with the Willmore energy. 
Problem 8.2 Given an $N$-graph $G$ with assigned angles we consider the minimization problem

$$
\inf \left\{\mathcal{W}(\mathcal{N}) \mid \mathcal{N}=(G, \Gamma) \in \mathcal{C}_{\text {Reg }} \text { with } \ell\left(\mathcal{N}^{1}\right), \ldots \ell\left(\mathcal{N}^{N}\right) \text { fixed }\right\}
$$

It is easy to prove that Problem 8.2 admits a minimizer in the class of regular networks by a direct method in the Calculus of Variations, as we shall now briefly sketch.

Consider a minimizing sequence of networks $\left\{\mathcal{N}_{n}\right\}_{n \in \mathbb{N}}$ composed of curves $\gamma_{n}^{i}$. Combining the bounds (3.3), (3.4) and (3.5) together with the fact that the length of each curve is fixed, we get that up to subsequence each $\gamma_{n}^{i}$ converges to a regular curve $\gamma_{\infty}^{i}$ weakly in $H^{2}$. The limit networks satisfies the angle condition in the sense of Definition 2.11 thanks to the strong $C^{1}$ convergence. Moreover the functional $\mathcal{W}$ is clearly lower semicontinuous.

Notice that fixing the length of each curve avoids any form of degeneracy of the limit networks and makes the question on existence of minimizers trivial.

Acknowledgements The research of the second and of the third author has been partially supported by INdAM - GNAMPA Project 2019 "Problemi geometrici per strutture singolari" (Geometric problems for singular structures). This project has received funding from the European Research Council (ERC) under the European Union's Horizon 2020 research and innovation programme under grant agreement No. 757254 (SINGULARITY).

Funding Open access funding provided by Università di Pisa within the CRUI-CARE Agreement.

Open Access This article is licensed under a Creative Commons Attribution 4.0 International License, which permits use, sharing, adaptation, distribution and reproduction in any medium or format, as long as you give appropriate credit to the original author(s) and the source, provide a link to the Creative Commons licence, and indicate if changes were made. The images or other third party material in this article are included in the article's Creative Commons licence, unless indicated otherwise in a credit line to the material. If material is not included in the article's Creative Commons licence and your intended use is not permitted by statutory regulation or exceeds the permitted use, you will need to obtain permission directly from the copyright holder. To view a copy of this licence, visit http://creativecommons.org/licenses/by/4.0/.

\section{A Critical Points}

We want now to derive the Euler Lagrange equations satisfied by regular critical points of the functional $\mathcal{E}$.

Let $\mathcal{N}$ be a regular network whose curves are parametrized by arclength by $\gamma^{i}$ : $\left[0, \ell\left(\gamma^{i}\right)\right] \rightarrow \mathbb{R}^{2}$. We denote by $s$ the arclength parameter of $\gamma^{i}$. We recall that $\partial_{s} \gamma=\tau$ and $\partial_{s} \tau=\vec{k}$. For sake of notation we introduce the operator $\partial_{s}^{\perp}$ that acts on a vector field $\varphi$ giving the normal component of $\partial_{s} \varphi$ along the curve $\gamma$, that is

$$
\partial_{s}^{\perp} \varphi=\partial_{s} \varphi-\left\langle\partial_{s} \varphi, \partial_{s} \gamma\right\rangle \partial_{s} \gamma
$$

Similarly we call $\partial_{s}^{\|} \varphi:=\left\langle\partial_{s} \varphi, \partial_{s} \gamma\right\rangle \partial_{s} \gamma=\left\langle\partial_{s} \varphi, \tau\right\rangle \tau$. In $\mathbb{R}^{2}$ it holds that $\partial_{s}^{\perp} \varphi=$ $\left\langle\partial_{s} \varphi, \nu\right\rangle \nu$, where $\nu$ is the counterclockwise rotation of $\partial_{s} \gamma$ by an angle equal to $\frac{\pi}{2}$. 
We compute a "regular" variation $\mathcal{N}_{\varepsilon}$ of $\mathcal{N}$. Each curve of $\mathcal{N}$ is parametrized by arclength by $\gamma^{i}$. Let us consider $\varepsilon \in \mathbb{R}$ and smooth functions $\psi^{i}:\left[0, \ell\left(\gamma^{i}\right)\right] \rightarrow \mathbb{R}^{2}$. This defines the variation $\gamma_{\varepsilon}^{i}:\left[0, \ell\left(\gamma^{i}\right)\right] \rightarrow \mathbb{R}^{2}$ of a curve $\gamma^{i}$ by setting $\gamma_{\varepsilon}^{i}:=\gamma^{i}+\varepsilon \psi^{i}$. Observe that the variation $\gamma_{\varepsilon}^{i}$ is not necessarily parametrized by arclength. However, for $\varepsilon \in\left(-\varepsilon_{0}, \varepsilon_{0}\right)$ with $\varepsilon_{0}>0$ sufficiently small, $\gamma_{\varepsilon}^{i}$ is a regular curve with tangent vector $\tau_{\varepsilon}^{i}=\frac{\partial_{s} \gamma^{i}+\varepsilon \partial_{s} \psi^{i}}{\left|\partial_{s} \gamma^{i}+\varepsilon \partial_{s} \psi^{i}\right|}$.

Consider a junction of order $m$ of $\mathcal{N}$, so that

$$
\gamma^{i_{1}}\left(z_{1}\right)=\ldots=\gamma^{i_{m}}\left(z_{m}\right)
$$

with $\left(z_{1}, i_{1}\right), \ldots,\left(z_{m}, i_{m}\right) \in\left\{0, \ell\left(\gamma^{i_{1}}\right), \ldots, \ell\left(\gamma^{i_{m}}\right)\right\} \times\{1, \ldots, N\}$ all distinct. The scalar product of unit tangent vectors is given by

$$
\left\langle\tau^{i_{1}}\left(z_{1}\right), \tau^{i_{2}}\left(z_{2}\right)\right\rangle=c^{1,2}, \ldots,\left\langle\tau^{i_{m-1}}\left(z_{m-1}\right), \tau^{i_{m}}\left(z_{m}\right)\right\rangle=c^{m-1, m} .
$$

To generate admissible competitors for the Problem 2.13 for any $\varepsilon$ small enough, we need to require that

$$
\gamma_{\varepsilon}^{i_{1}}\left(z_{1}\right)=\ldots=\gamma_{\varepsilon}^{i_{m}}\left(z_{m}\right)
$$

together with the fact that the angle condition is preserved, that is to say that for every $i, j, z, w$ such that $\gamma^{i}(z)=\gamma^{j}(w)$ it holds that

$$
\frac{\mathrm{d}}{\mathrm{d} \varepsilon}\left\langle\tau_{\varepsilon}^{i}(z), \tau_{\varepsilon}^{j}(w)\right\rangle=0
$$

for every $\varepsilon \in\left(-\varepsilon_{0}, \varepsilon_{0}\right)$.

First this implies that the variation fields $\psi^{i}$ satisfy that

$$
\psi^{i_{1}}\left(z_{1}\right)=\ldots=\psi^{i_{m}}\left(z_{m}\right)
$$

Secondly, writing

$$
\begin{aligned}
\partial_{s} \psi^{i}=\partial_{s}^{\perp} \psi^{i}+\partial_{s}^{\|} \psi^{i} & =\left\langle\partial_{s} \psi^{i}, v^{i}\right\rangle v^{i}+\left\langle\partial_{s} \psi^{i}, \tau^{i}\right\rangle \tau^{i} \\
& =: \bar{\psi}_{s}^{i} \nu^{i}+\widetilde{\psi}_{s}^{i} \tau^{i},
\end{aligned}
$$

a direct calculation yields

$$
\begin{aligned}
0= & \frac{\mathrm{d}}{\mathrm{d} \varepsilon}\left\langle\tau_{\varepsilon}^{i}(z), \tau_{\varepsilon}^{j}(w)\right\rangle=\left\langle\left.\frac{\partial_{s} \psi^{i}-\left\langle\partial_{s} \psi^{i}, \tau_{\varepsilon}^{i}\right\rangle \tau_{\varepsilon}^{i}}{\left|\partial_{s} \gamma^{i}+\varepsilon \partial_{s} \psi^{i}\right|}\right|_{z}, \tau_{\varepsilon}^{j}(w)\right\rangle \\
& +\left\langle\tau_{\varepsilon}^{i}(z),\left.\frac{\partial_{s} \psi^{i}-\left\langle\partial_{s} \psi^{i}, \tau_{\varepsilon}^{i}\right\rangle \tau_{\varepsilon}^{i}}{\left|\partial_{s} \gamma^{i}+\varepsilon \partial_{s} \psi^{i}\right|}\right|_{w}\right\rangle
\end{aligned}
$$


Evaluating at $\varepsilon=0$ we get

$$
\begin{aligned}
0 & =\left\langle\partial_{s}^{\perp} \psi^{i}(z), \tau^{j}(w)\right\rangle+\left\langle\tau^{i}(z), \partial_{s}^{\perp} \psi^{j}(w)\right\rangle \\
& =\bar{\psi}_{s}^{i}(z)\left\langle v^{i}(z), \tau^{j}(w)\right\rangle+\bar{\psi}_{s}^{j}(w)\left\langle\tau^{i}(z), v^{j}(w)\right\rangle \\
& =\bar{\psi}_{s}^{i}(z)\left\langle v^{i}(z), \tau^{j}(w)\right\rangle-\bar{\psi}_{s}^{j}(w)\left\langle v^{i}(z), \tau^{j}(w)\right\rangle .
\end{aligned}
$$

Therefore as a second requirement on the fields $\psi^{i}$ we impose that

$$
\bar{\psi}_{s}^{i_{1}}\left(z_{1}\right)=\ldots=\bar{\psi}_{s}^{i_{m}}\left(z_{m}\right)
$$

Definition A.1 Consider a regular network $\mathcal{N}=(G, \Gamma)$ composed of the curves $\gamma^{i}$ parametrized by arclength with $i \in\{1, \ldots, N\}, \varepsilon \in \mathbb{R}$ and smooth functions $\psi^{i}$ : $\left[0, \ell\left(\gamma^{i}\right)\right] \rightarrow \mathbb{R}^{2}$. We say that $\mathcal{N}_{\varepsilon}$ composed of the curves $\gamma_{\varepsilon}^{i}=\gamma^{i}+\varepsilon \psi^{i}$ is a regular variation of $\mathcal{N}$ if the functions $\psi^{i}$ satisfy the properties (A.1) and (A.2).

Definition A.2 A regular network $\mathcal{N}=(G, \Gamma)$ is a critical point for the functional $\mathcal{E}$ if for every regular variation $\mathcal{N}_{\varepsilon}$ of $\mathcal{N}$ it holds

$$
\frac{\mathrm{d}}{\mathrm{d} \varepsilon} \mathcal{E}\left(\mathcal{N}_{\varepsilon}\right)_{\mid \varepsilon=0}=0
$$

We recall that the oriented curvature of a planar curve $\gamma$ is defined as the scalar $k$ such that $\vec{k}=k v$ where the unit normal vector $v$ is the counterclockwise rotation of $\frac{\pi}{2}$ of the unit tangent vector $\tau$ to the curve $\gamma$.

Proposition A.3 Let $\mathcal{N}=(G, \Gamma)$ be a critical point for $\mathcal{E}$. Then the arclength parametrization $\gamma^{i}:\left[0, \ell\left(\gamma^{i}\right)\right] \rightarrow \mathbb{R}^{2}$ of any curve of $\mathcal{N}$ is real analytic and satisfies the equation

$$
2\left(\partial_{s}^{\perp}\right)^{2} \vec{k}^{i}+\left|\vec{k}^{i}\right|^{2} \vec{k}^{i}-\vec{k}^{i}=0 \quad \text { on }\left(0, \ell\left(\gamma^{i}\right)\right),
$$

or, equivalently, in terms of the oriented curvature

$$
2 \partial_{s}^{2} k^{i}+\left(k^{i}\right)^{3}-k^{i}=0 \quad \text { on }\left(0, \ell\left(\gamma^{i}\right)\right)
$$

Also, the curves satisfy the following boundary conditions. If $p=\pi\left(z_{1}, i_{1}\right)=\cdots=$ $\pi\left(z_{m}, i_{m}\right)$ is a junction of order $m$ of $\mathcal{N}$, then

$$
\begin{gathered}
\sum_{\substack{\left(z_{j}, i_{j}\right) \in \pi^{-1}(p): \\
z_{j}=\ell\left(\gamma^{i j}\right)}} k^{i_{j}}\left(\ell\left(\gamma^{i_{j}}\right)\right) \\
=\sum_{\substack{\left(z_{j}, i_{j}\right) \in \pi^{-1}(p) \\
z_{j}=0}} k^{i_{j}}(0),
\end{gathered}
$$




$$
\begin{aligned}
& \left.\sum_{\substack{\left(z_{j}, i_{j}\right) \in \pi^{-1}(p): \\
z_{j}=\ell\left(\gamma^{i} j\right)}}\left[2 \partial_{s}^{\perp} \vec{k}^{i_{j}}+\left(k^{i_{j}}\right)^{2} \tau^{i_{j}}-\tau^{i_{j}}\right]\right|_{\ell\left(\gamma^{i_{j}}\right)} \\
& =\left.\sum_{\substack{\left(z_{j}, i_{j}\right) \in \pi^{-1}(p) \\
z_{j}=0}}\left[2 \partial_{s}^{\perp} \vec{k}^{i_{j}}+\left(k^{i_{j}}\right)^{2} \tau^{i_{j}}-\tau^{i_{j}}\right]\right|_{0} .
\end{aligned}
$$

Remark A.4 Let us observe that the boundary conditions (A.4) and (A.5) do not depend on the parametrizations of the curves $\gamma^{i}$. More precisely, even if such boundary conditions are expressed in terms of quantities evaluated at 0 or at $\ell\left(\gamma^{i}\right)$, if a curve $\gamma^{j}$ is reparametrized into the new arclength parametrized immersion $\tilde{\gamma}^{j}(t)=\gamma^{j}\left(\ell\left(\gamma^{j}\right)-t\right)$, then

$$
\begin{gathered}
\tilde{k}^{j}\left(\ell\left(\gamma^{j}\right)\right)=-k^{j}(0), \quad \tilde{k}^{j}(0)=-k^{j}\left(\ell\left(\gamma^{j}\right)\right), \\
{\left.\left[2 \partial_{\tilde{s}^{j}}^{\perp} \tilde{\vec{k}}^{j}+\left(\tilde{k}^{j}\right)^{2} \tilde{\tau}^{j}-\tilde{\tau}^{j}\right]\right|_{\ell\left(\gamma^{j}\right)}=-\left.\left[2 \partial_{s^{j}}^{\perp} \vec{k}^{j}+\left(k^{j}\right)^{2} \tau^{j}-\tau^{j}\right]\right|_{0},} \\
{\left.\left[2 \partial_{\tilde{s}^{j}}^{\perp} \overrightarrow{\tilde{k}}^{j}+\left(\tilde{k}^{j}\right)^{2} \tilde{\tau}^{j}-\tilde{\tau}^{j}\right]\right|_{0}=-\left.\left[2 \partial_{s^{j}}^{\perp} \vec{k}^{j}+\left(k^{j}\right)^{2} \tau^{j}-\tau^{j}\right]\right|_{\ell\left(\gamma^{j}\right)},}
\end{gathered}
$$

where symbols with the tilde identify the obvious geometric quantities in terms of the parametrization $\tilde{\gamma}^{k}$.

Proof of Proposition A.3 In order to calculate the first variation of the functional, we can fix a junction $p$ of $\mathcal{N}$ of order $m$ and consider a regular variation given by fields $\psi^{i_{j}}$ for $j=1, \ldots, m$ such that: if $\pi\left(z_{j}, i_{j}\right)=p$ but $\pi\left(1-z_{j}, i_{j}\right) \neq p$, then $\psi^{i_{j}} \equiv 0$ in a neighborhood of $1-z_{j}$. By direct computations (for the details for example see [22]) one shows that the curves are of class $C^{\infty}$ and they satisfy the first variation formula

$$
\begin{aligned}
\left.\frac{\mathrm{d}}{\mathrm{d} \varepsilon} \mathcal{E}\left(\mathcal{N}_{\varepsilon}\right)\right|_{\varepsilon=0}= & \sum_{j=1}^{m} \int_{\gamma^{i_{j}}}\left\langle 2\left(\partial_{s}^{\perp}\right)^{2} \vec{k}^{i_{j}}+\left|\vec{k}^{i_{j}}\right|^{2} \vec{k}^{i_{j}}-\vec{k}^{i_{j}}, \psi^{i_{j}}\right\rangle \mathrm{d} s \\
& +\sum_{j=1}^{m}\left[\left.2\left\langle\vec{k}^{i_{j}}, \partial_{s} \psi^{i_{j}}\right\rangle\right|_{0} ^{\ell\left(\gamma^{i_{j}}\right)}\right. \\
& \left.+\left.\left\langle-2 \partial_{s}^{\perp} \vec{k}^{i_{j}}-\left|\vec{k}^{i_{j}}\right|^{2} \tau^{i_{j}}+\tau^{i_{j}}, \psi^{i_{j}}\right\rangle\right|_{0} ^{\ell\left(\gamma^{i_{j}}\right)}\right]=0 .
\end{aligned}
$$

This immediately leads to the interior equations (A.3).

In order to get the boundary conditions, we can first set

$$
\psi^{i_{j}}(0)=\psi^{i_{j}}\left(\ell\left(\gamma^{i_{j}}\right)\right)=0
$$


so that

$$
\left.\sum_{j=1}^{m} \bar{\psi}_{s}^{i} k^{i_{j}}\right|_{0} ^{\ell\left(\gamma^{i} j\right)}=0
$$

and by (A.2) we get the first boundary condition (A.4) Similarly, letting $\bar{\psi}_{s}^{i}(0)=$ $\bar{\psi}_{s}^{i}\left(\ell\left(\gamma^{i_{j}}\right)\right)=0$ and using (A.1), the second boundary condition (A.5) is achieved.

Now we prove that each $\gamma^{i}$ is an analytic curve. Fix $i=1$, and suppose that $\pi(0,1)$ is a junction of order $m$. The first boundary condition equation gives that

$$
k^{1}(0)=\sum_{\substack{\left(z_{j}, i_{j}\right) \in \pi^{-1}(p): \\ z_{j}=\ell\left(\gamma^{i j}\right)}} k^{i_{j}}\left(\ell\left(\gamma^{i_{j}}\right)\right)-\sum_{\substack{\left(z_{j}, i_{j}\right) \in \pi^{-1}(p): \\ z_{j}=0, j \neq 1}} k^{i_{j}}(0)=: C_{1} .
$$

While, since $\left\langle\partial_{s}^{\perp} \vec{k}^{1}(0), v^{1}(0)\right\rangle=\partial_{s} k^{1}(0)$, by multiplying the second boundary condition equation by $v^{1}(0)$ we can write that

$$
\begin{aligned}
\partial_{s} k^{1}(0)= & \frac{1}{2}\left\langle\left.\sum_{\substack{\left(z_{j}, i_{j}\right) \in \pi^{-1}(p): \\
z_{j}=\ell\left(\gamma^{i j}\right)}}\left[2 \partial_{s}^{\perp} \vec{k}^{i_{j}}+\left(k^{i_{j}}\right)^{2} \tau^{i_{j}}-\tau^{i_{j}}\right]\right|_{\ell\left(\gamma^{i_{j}}\right)}+\right. \\
& \left.-\left.\sum_{\substack{\left(z_{j}, i_{j}\right) \in \pi^{-1}(p): \\
z_{j}=0, j \neq 1}}\left[2 \partial_{s}^{\perp} \vec{k}^{i_{j}}+\left(k^{i_{j}}\right)^{2} \tau^{i_{j}}-\tau^{i_{j}}\right]\right|_{0}, v^{1}(0)\right\rangle=: C_{2} .
\end{aligned}
$$

Therefore a direct application of the Cauchy-Kovaleskaya Theorem (see for instance $[12$, p. 240]) on the Cauchy problem

$$
\left\{\begin{array}{l}
2 \partial_{s}^{2} k^{1}+\left(k^{1}\right)^{3}-k^{1}=0 \quad \text { on }[0, \varepsilon), \\
k^{1}(0)=C_{1}, \\
\partial_{s} k^{1}(0)=C_{2},
\end{array}\right.
$$

gives that $k^{1}$ is real analytic on some interval $[0, \varepsilon)$. Analogously, the same holds on $\left(\ell\left(\gamma^{1}\right)-\varepsilon, \ell\left(\gamma^{1}\right)\right]$, and therefore $k^{1}$ is real analytic. Writing $\tau^{1}(s)=$ $(\cos (\theta(s)), \sin (\theta(s))$ we get that

$$
\partial_{s} \tau^{1}=(-\sin (\theta), \cos (\theta)) \partial_{s} \theta,
$$

so that

$$
k^{1}(s)=\partial_{s} \theta(s)
$$

Hence $\theta$ is analytic, that implies that $\partial_{s} \gamma^{1}=\tau^{1}$ is analytic, and so is $\gamma^{1}$.

By the arbitrary of the choice of $\gamma^{1}$, the result follows for each $\gamma^{i}$. 


\section{References}

1. Abate, M., Tovena, F.: Curves and surfaces, Unitext, vol. 55, Springer, Milan, Translated from the 2006 Italian original by Daniele A. Gewurz (2012)

2. Alessandroni, R., Kuwert, E.: Local solutions to a free boundary problem for the Willmore functional, Calc. Var. Partial Differ. Equ. 55(2), Art. 24, 29 (2016)

3. Barrett, J.W., Garcke, H., Nürnberg, R.: Elastic flow with junctions: variational approximation and applications to nonlinear splines, Math. Models Methods Appl. Sci. 22(11), 1250037, 57 (2012)

4. Bellettini, G., Mugnai, L.: Characterization and representation of the lower semicontinuous envelope of the elastica functional. Ann. Inst. H. Poincaré Anal. Non Linéaire 21(6), 839-880 (2004)

5. Bellettini, G., Mugnai, L.: A varifolds representation of the relaxed elastica functional. J. Convex Anal. 14(3), 543-564 (2007)

6. Bellettini, G., Paolini, M.: Variational properties of an image segmentation functional depending on contours curvature. Adv. Math. Sci. Appl. 5(2), 681-715 (1995)

7. Bellettini, G., Dal Maso, G., Paolini, M.: Semicontinuity and relaxation properties of a curvature depending functional in 2D. Ann. Scuola Norm. Sup. Pisa Cl. Sci. (4) 20(2), 247-297 (1993)

8. Dall'Acqua, A., Novaga, M., Pluda, A.: Minimal elastic networks, to appear: Indiana Univ. Math. J., preprint arXiv: 1712.09589

9. Dondl, P.W., Lemenant, A., Wojtowytsch, S.: Phase field models for thin elastic structures with topological constraint. Arch. Rational Mech. Anal. 223(2), 693-736 (2017)

10. Dall'Acqua, A., Pluda, A.: Some minimization problems for planar networks of elastic curves. Geom. Flows 2(1), 105-124 (2017)

11. Deckelnick, K., Grunau, H.-C., Röger, M.: Minimising a relaxed Willmore functional for graphs subject to boundary conditions. Interfaces Free Bound. 19(1), 109-140 (2017)

12. Garcke, H., Menzel, J., Pluda, A.: Long time existence of solutions to an elastic flow of networks, preprint (2019)

13. Garcke, H., Menzel, J., Pluda, A.: Willmore flow of planar networks. J. Differ. Equ. 266(4), 2019-2051 (2019)

14. Lawrence, C.: Partial differential equations, Graduate Studies in Mathematics, vol. 19. American Mathematical Society, Providence, RI (1998)

15. Masnou, S., Nardi, G.: A coarea-type formula for the relaxation of a generalized elastica functional. J. Convex Anal. 20(3), 617-653 (2013)

16. Masnou, S., Nardi, G.: Gradient Young measures, varifolds, and a generalized Willmore functional. Adv. Calc. Var. 6(4), 433-482 (2013)

17. Menzel, J.: PhD thesis: boundary value problems for generalized willmore flows, Universität Regensburg (in preparation)

18. Mumford, D.: Elastica and Computer Vision, pp. 491-506. Springer, New York (1994)

19. Pozzetta, M.: On the Plateau-Douglas problem for the Willmore energy of surfaces with planar boundary curves, To appear: ESAIM:COCV (2020)

20. Pozzetta, M.: A varifold perspective on the p-elastic energy of planar sets. J. Convex Anal. 27(3), 845-879 (2020)

21. Schätzle, R.: The Willmore boundary problem. Calc. Var. Partial Differ. Equ. 37(3-4), 275-302 (2010)

22. Truesdell, C.A.: The influence of elasticity on analysis: the classic heritage. Bull. Am. Math. Soc. (N.S.) 9(3), 293-310 (1983)

Publisher's Note Springer Nature remains neutral with regard to jurisdictional claims in published maps and institutional affiliations. 